\title{
INERTIA GROUPS OF A TORIC DELIGNE-MUMFORD STACK, FAKE WEIGHTED PROJECTIVE STACKS, AND LABELED SHEARED SIMPLICES
}

\author{
REBECCA GOLDIN, MEGUMI HARADA, \\ DAVID JOHANNSEN AND DEREK KREPSKI
}

\begin{abstract}
This paper determines the inertia groups (isotropy groups) of the points of a toric Deligne-Mumford stack $[Z / G]$ (considered over the category of smooth manifolds) that is realized from a quotient construction using a stacky fan or stacky polytope. The computation provides an explicit correspondence between certain geometric and combinatorial data. In particular, we obtain a computation of the connected component of the identity element $G_{0} \subset G$ and the component group $G / G_{0}$ in terms of the underlying stacky fan, enabling us to characterize the toric DM stacks which are global quotients. As another application, we obtain a characterization of those stacky polytopes that yield stacks equivalent to weighted projective stacks and, more generally, to 'fake' weighted projective stacks. Finally, we illustrate our results in detail in the special case of labeled sheared simplices, where explicit computations can be made in terms of the facet labels.
\end{abstract}

Introduction. Toric varieties have been studied for over 35 years. They provide an elementary but illustrative class of examples in algebraic geometry, while also offering insight into related fields such as integrable systems and combinatorics, where the corresponding combinatorial object is a fan. In their foundational paper [5], Borisov, Chen and Smith introduce the notion of a stacky fan, the combinatorial data from which one constructs toric Deligne-Mumford (DM) stacks, which are the stack-theoretic analogues of classical toric vari-

2010 AMS Mathematics subject classification. Primary 14M25, 57R18, Secondary $14 \mathrm{~L} 24$.

Keywords and phrases. Stacky fan, toric Deligne-Mumford stack, inertia group, weighted projective spaces.

The first author is partially supported by NSF Disciplinary grant Nos. 1201458 and 202726. The second author is partially supported by an NSERC Discovery grant, an Ontario Ministry of Research and Innovation Early Researcher Award and a Canada Research Chair (Tier 2) award.

Received by the editors on June 29, 2014. 
eties. When the corresponding fan is polytopal, classical toric varieties have been studied from the perspectives of both algebraic and symplectic geometry. Similarly, when the underlying fan of a stacky fan is polytopal, a toric DM stack admits a description in the language of symplectic geometry via the combinatorial data of a stacky polytope introduced by Sakai [26]. (In the symplectic-geometric context-and particularly in this manuscript-stacks are considered over the category Diff of smooth manifolds.) This subfamily of toric DM stacks can be viewed as a generalization of Lerman and Tolman's toric orbifolds associated to labeled polytopes [20] (cf., subsection 1.2 for details), which are in turn a generalization of the Delzant polytopes that classify symplectic toric manifolds. Thus, toric DM stacks are a generalization of smooth toric varieties to not necessarily effective orbifold toric varieties (including weighted projective spaces), and provide a fertile ground for exploration of stacks via this large class of examples.

The exposition in this article is intended to be accessible to a wide audience, including researchers who are not experts in this area. The tools we develop allow one to concretely compute the isotropy groups of toric DM stacks without reference to much stack-theoretic machinery. Moreover, we include many detailed examples illustrating our results.

The mathematical contributions of this manuscript are as follows. We first describe in Theorem 2.2, Proposition 2.4 and Proposition 2.15 an explicit computation of the isotropy groups of toric DM stacks, realized as quotient stacks $[Z / G]$ for appropriate space $Z$ and abelian Lie group $G$, in terms of the combinatorial data (i.e., stacky fan) determining the toric DM stack.

Second, as an application of our description of isotropy groups of toric DM stacks, we give a computation of the connected component of the identity element $G_{0} \subset G$ and the component group $G / G_{0}$ in terms of the underlying stacky fan (Proposition 2.11, Lemma 2.8, Proposition 2.17). To place this computation into context, recall that a stack is called a global quotient if it is equivalent to a quotient stack $[M / \Lambda]$ where $\Lambda$ is a finite group acting on a manifold $M$. Stack invariants of global quotients are simpler to compute than general stacks. Thus, given a stack $\mathcal{X}$, it is an interesting problem to determine whether or not it is a global quotient. The case of toric DM stacks is discussed in [14], where it is shown that a toric DM stack is a global quotient if and only if the restriction of the $G$-action on $Z$ to the 
connected component of the identity $G_{0} \subset G$ is a free action. Moreover, in this case, one may choose the finite group to be $\Lambda=G / G_{0}$, acting on the quotient $M=Z / G_{0}$, which is indeed a manifold, provided $G_{0}$ acts freely on $Z$ (so $\left[Z / G_{0}\right]$ is the universal cover, in the sense of stacks, of $[Z / G])$. In this manuscript, our computation of isotropy groups leads to a characterization of those toric DM stacks that are (stacks equivalent to) global quotients of a finite group action and to a description of its universal cover (cf., subsection 2.2).

Our third set of results concern weighted projective stacks (respectively, fake weighted projective stacks), which are natural stacktheoretic analogues of the classical weighted projective spaces (respectively, fake weighted projective spaces as considered in $[\mathbf{6}, \mathbf{1 7}])$. These form a rich class of examples that have been studied extensively both as stacks and as orbifolds (e.g., see $[4,16,21]$, among others). As another application of our computation of isotropy groups, in Proposition 3.2 (respectively, Proposition 3.4) we obtain an exact characterization of those stacky polytopes which yield (stacks equivalent to) weighted projective stacks (respectively, fake weighted projective stacks).

Finally, in Section 4, we introduce a class of labeled polytopes, which we call labeled sheared simplices. These are labeled simplices with all facets but one lying on coordinate hyperplanes. In this special case, we concretely illustrate the aforementioned results in terms of the facet labels.

1. Preliminaries. In this section, we recall some background regarding stacky fans and polytopes and their associated toric DeligneMumford stacks. We assume some familiarity with stacks, in particular their use in modeling group actions on manifolds and orbifolds. We refer the reader to, e.g., $[\mathbf{7}, \mathbf{8}]$ and the references therein for basic definitions and ideas in the theory of stacks. Within the field of algebraic geometry there is now an extensive literature on (algebraic) stacks (see, e.g., the informal guide [1]), but in other categories (e.g., Diff or Top) the literature continues to develop. The present authors learned a great deal from the unpublished (in-progress) notes $[\mathbf{3}]$ as well as $[\mathbf{1 8}, \mathbf{2 2}]$.

The stacks appearing in this paper are quotient stacks over Diff associated to smooth, proper, locally-free Lie group actions on manifolds. Such quotient stacks are Deligne-Mumford (i.e., stacks that admit a presentation by a proper étale Lie groupoid, cf., [19, Theorem 2.4]) 
and therefore model smooth orbifolds. Often in the algebraic literature, the term orbifold is used for DM stacks with trivial generic stabilizer (e.g., as in [9]), what we shall refer to as an effective orbifold.

The class of DM stacks we work with are toric DM stacks, arising from the combinatorial data of a stacky fan [5]. Our original motivation was to work instead with stacky polytopes, the symplectic counterparts of stacky fans, which give rise to symplectic toric DM stacks [26]. These offer a modern perspective on the symplectic toric orbifolds of Lerman and Tolman [20] constructed from labeled polytopes (see subsection 1.2 below). However, since our results do not depend on (or make use of) the symplectic structure that results from this perspective, we choose to mainly work with stacky fans.

1.1. Stacky fans and polytopes. Mainly to establish notation, we briefly recall some basic definitions of the combinatorial data appearing in the above discussion. We use $(-)^{\star}$ to denote the functor $\operatorname{Hom}_{\mathbb{Z}}(-, \mathbb{Z})$ or $\operatorname{Hom}_{\mathbb{R}}(-, \mathbb{R})$; it should be clear from the context which one is meant. Let $\mathbb{T}$ denote the group of units $\mathbb{C}^{\times}$, and $\mu_{k} \subset \mathbb{T}$ the cyclic group of $k$ th roots of unity. Let $\left\{e_{1}, \ldots, e_{n}\right\}$ be the standard basis vectors in $\mathbb{Z}^{n} \subset \mathbb{R}^{n}$.

Definition $1.1([5])$. A stacky fan is a triple $(N, \Sigma, \beta)$ consisting of a rank $d$ finitely generated Abelian group $N$, a rational simplicial fan $\Sigma$ in $N \otimes \mathbb{R}$ with rays $\rho_{1}, \ldots, \rho_{n}$ and a homomorphism $\beta: \mathbb{Z}^{n} \rightarrow N$ satisfying:

(i) the rays $\rho_{1}, \ldots, \rho_{n}$ span $N \otimes \mathbb{R}$, and

(ii) for $1 \leq j \leq n, \beta\left(e_{j}\right) \otimes 1$ is on the ray $\rho_{j}$.

Given a polytope $\Delta \subseteq \mathbb{R}^{d}$, recall that the corresponding fan $\Sigma=$ $\Sigma(\Delta)$ is obtained by setting the one-dimensional cones $\Sigma^{(1)}$ to be the positive rays spanned by the inward-pointing normals to the facets of $\Delta$; a subset $\sigma$ of these rays is a cone in $\Sigma$ precisely when the corresponding facets intersect nontrivially in $\Delta$. Observe that, under this correspondence, facets intersecting in a vertex of $\Delta$ yield maximal cones (with respect to inclusion) in $\Sigma(\Delta)$.

Definition $1.2([26])$. A stacky polytope is a triple $(N, \Delta, \beta)$ consisting of a rank $d$ finitely generated Abelian group $N$, a simple polytope $\Delta$ in 
$(N \otimes \mathbb{R})^{\star}$ with $n$ facets $F_{1}, \ldots, F_{n}$ and a homomorphism $\beta: \mathbb{Z}^{n} \rightarrow N$ satisfying:

(i) the cokernel of $\beta$ is finite, and

(ii) for $1 \leq j \leq n, \beta\left(e_{j}\right) \otimes 1$ in $N \otimes \mathbb{R}$ is an inward pointing normal to the facet $F_{j}$.

Condition (ii) above implies that the polytope $\Delta$ in Definition 1.2 is a rational polytope. Also, from the preceding discussion it follows immediately that the data of a stacky polytope $(N, \Delta, \beta)$ specifies the data of a stacky fan by the correspondence $(N, \Delta, \beta) \mapsto(N, \Sigma(\Delta), \beta)$. Indeed, $\Delta$ is simple if and only if $\Sigma(\Delta)$ is simplicial. Moreover, the fan $\Sigma(\Delta)$ is rational by condition 1.2 (ii). Finally, $(N, \Delta, \beta)$ satisfies conditions (i) and (ii) of Definition 1.2 if and only if $(N, \Sigma(\Delta), \beta)$ satisfies conditions (i) and (ii) of Definition 1.1.

The extra information encoded in a stacky polytope $(N, \Delta, \beta)$ (compared with the stacky fan $(N, \Sigma(\Delta), \beta)$ ) results in a symplectic structure on the associated toric DM stack. Given a presentation of a rational polytope $\Delta$ as the intersection of half-spaces

$$
\Delta=\bigcap_{i=1}^{n}\left\{x \in(N \otimes \mathbb{R})^{\star} \mid x\left(\beta\left(e_{i}\right) \otimes 1\right) \geq-c_{i}\right\}
$$

for some $c_{i} \in \mathbb{R}$ and where each $\beta\left(e_{i}\right) \otimes 1 \in N \otimes \mathbb{R}$ is the inward pointing normal to the facet $F_{i}$, the fan $\Sigma(\Delta)$ only retains the data of the positive ray spanned by the normals, and not the parameters $c_{i}$, which encode the symplectic structure on the resulting DM stack (see, [26] for details).

Recall (as in [5]) that, given a stacky fan $(N, \Sigma, \beta)$, the corresponding DM stack may be constructed as a quotient stack $\left[Z_{\Sigma} / G\right]$ as follows. As with classical toric varieties, the fan $\Sigma$ determines an ideal

$$
J(\Sigma)=\left\langle\prod_{\rho_{i} \not \subset \sigma} z_{i}: \sigma \in \Sigma\right\rangle \subset \mathbb{C}\left[z_{1}, \ldots, z_{n}\right] .
$$

Let $Z_{\Sigma}$ denote the complement $\mathbb{C}^{n} \backslash V(J(\Sigma))$ of the vanishing locus of $J(\Sigma)$. Next, we recall a certain group action on $Z_{\Sigma}$. 
Choose a free resolution

$$
0 \longrightarrow \mathbb{Z}^{\ell} \stackrel{Q}{\longrightarrow} \mathbb{Z}^{d+\ell} \longrightarrow N \longrightarrow 0
$$

of the $\mathbb{Z}$-module $N$, and let $B: \mathbb{Z}^{n} \rightarrow \mathbb{Z}^{d+\ell}$ be a lift of $\beta$. With these choices, define the dual group $\operatorname{DG}(\beta)=\left(\mathbb{Z}^{n+\ell}\right)^{\star} / \operatorname{im}[B Q]^{\star}$ where $[B Q]: \mathbb{Z}^{n+\ell}=\mathbb{Z}^{n} \oplus \mathbb{Z}^{\ell} \rightarrow \mathbb{Z}^{d+\ell}$ denotes the map whose restrictions to the first and second summands are $B$ and $Q$, respectively. Let $\beta^{\vee}:\left(\mathbb{Z}^{n}\right)^{\star} \rightarrow \operatorname{DG}(\beta)$ be the composition of the inclusion $\left(\mathbb{Z}^{n}\right)^{\star} \rightarrow\left(\mathbb{Z}^{n+\ell}\right)^{\star}$ (into the first $n$ coordinates) and the quotient map $\left(\mathbb{Z}^{n+\ell}\right)^{\star} \rightarrow \operatorname{DG}(\beta)$. Applying the functor $\operatorname{Hom}_{\mathbb{Z}}(-, \mathbb{T})$ to $\beta^{\vee}$ yields a homomorphism $G:=\operatorname{Hom}_{\mathbb{Z}}(\operatorname{DG}(\beta), \mathbb{T}) \rightarrow \mathbb{T}^{n}$, which defines a $G$-action on $\mathbb{C}^{n}$ that leaves $Z_{\Sigma} \subset \mathbb{C}^{n}$ invariant. Define $\mathcal{X}(N, \Sigma, \beta)=\left[Z_{\Sigma} / G\right]$. By [5, Proposition 3.2], $\mathcal{X}(N, \Sigma, \beta)$ is a DM stack. At times, we shall simply use the notation $\left[Z_{\Sigma} / G\right]$ to denote $\mathcal{X}(N, \Sigma, \beta)$.

The above construction was adapted to stacky polytopes by Sakai in [26]. As the reader may verify, the DM stack $\mathcal{X}(N, \Delta, \beta)$ obtained from a stacky polytope is a quotient stack obtained by symplectic reduction $\left[\mu^{-1}(\tau) / K\right]$ where $\mu^{-1}(\tau) \subset Z_{\Sigma(\Delta)} \subset \mathbb{C}^{n}$ is a certain level set of a moment map $\mu: \mathbb{C}^{n} \rightarrow \mathfrak{k}^{\star}$ for a Hamiltonian action of $K:=\operatorname{Hom}_{\mathbb{Z}}\left(\mathrm{DG}(\beta), S^{1}\right)$ on $\mathbb{C}^{n}$. By [26, Theorem 24], the quotient stacks $\left[Z_{\Sigma} / G\right]$ and $\left[\mu^{-1}(\tau) / K\right]$ are equivalent.

Example 1.3. Consider the stacky polytope $(N, \Delta, \beta)$, with $N=\mathbb{Z}^{2}$, $\Delta$ the simplex in $\mathbb{R}^{2} \cong(N \otimes \mathbb{R})^{*}$ given by the convex hull of $(0,0)$, $(0,1)$ and $(1,0)$, and $\beta: \mathbb{Z}^{3} \rightarrow N$ given by the matrix

$$
\beta=\left[\begin{array}{lll}
-2 & 3 & 0 \\
-2 & 0 & 5
\end{array}\right] \text {. }
$$

The corresponding stacky fan $(N, \Sigma, \beta)$ is then given by the same $N$ and $\beta$, and $\Sigma=\Sigma(\Delta)$ the fan dual to $\Delta$ (see Figure 1). A convenient way to represent the homomorphism $\beta$ is to use ray or facet labels (see subsection 1.2), as in Figure 1.

To compute the corresponding DM stack $\left[Z_{\Sigma} / G\right]$, note that $Z_{\Sigma}=$ $\mathbb{C}^{3} \backslash\{0\}$. We find $\operatorname{DG}(\beta)=\left(\mathbb{Z}^{3}\right)^{\star} / \operatorname{im} \beta^{\star} \cong \mathbb{Z}$, where the isomorphism may be chosen as $\bar{f}([a, b, c])=15 a+10 b+6 c$. Therefore, $G=$ $\operatorname{Hom}(\mathrm{DG}(\beta), \mathbb{T}) \cong \mathbb{T}$. Since the map $\beta^{\vee}$ is simply the projection $f:\left(\mathbb{Z}^{3}\right)^{\star} \rightarrow \operatorname{DG}(\beta) \cong \mathbb{Z}$, where $f(a, b, c)=15 a+10 b+6 c$, the homomorphism $G \rightarrow \mathbb{T}^{3}$ induced by $\beta^{\vee}$ is then $t \mapsto\left(t^{15}, t^{10}, t^{6}\right)$. 


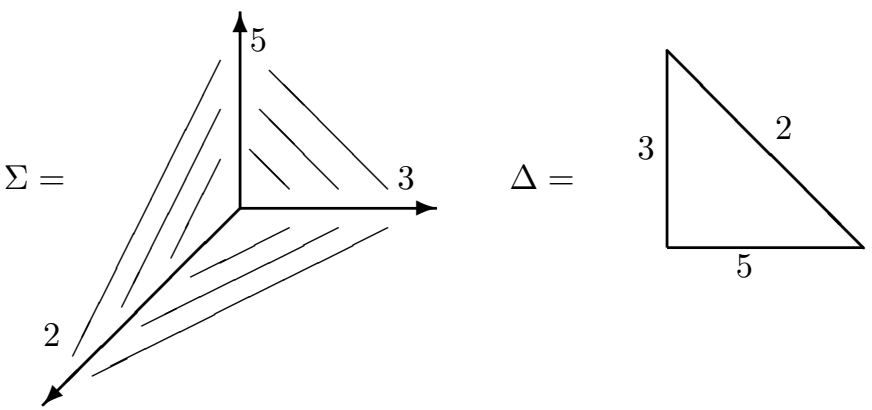

Figure 1. A polytope $\Delta$ and its dual fan $\Sigma=\Sigma(\Delta)$. The labels on the facets of $\Delta$ (respectively, ray generators of $\Sigma$ ) encode the homomorphism $\beta: \mathbb{Z}^{3} \rightarrow N$.

It follows that the corresponding stack is equivalent to a weighted projective stack, $\mathcal{P}(15,10,6)$.

We modify the above example to illustrate the construction for a $\mathbb{Z}$-module $N$ with torsion.

Example 1.4. Let $N=\mathbb{Z}^{2} \oplus \mathbb{Z} / 2 \mathbb{Z}$, and let $\Sigma$ be the fan in Figure 1 . Set $\beta: \mathbb{Z}^{3} \rightarrow N$ to be

$$
\beta(x, y, z)=(-2 x+3 y,-2 x+5 z, x+y+z \bmod 2) .
$$

As in Example 1.3, $Z_{\Sigma}=\mathbb{C}^{3} \backslash\{0\}$. To compute $G$, we choose the resolution $0 \rightarrow \mathbb{Z} \stackrel{Q}{\rightarrow} \mathbb{Z}^{3} \rightarrow N \rightarrow 0$ with $Q=\left[\begin{array}{lll}0 & 0 & 2\end{array}\right]^{T}$, and choose $B$ so that

$$
[B Q]=\left[\begin{array}{llll}
-2 & 3 & 0 & 0 \\
-2 & 0 & 5 & 0 \\
-1 & 1 & 1 & 2
\end{array}\right] \text {. }
$$

Therefore, $\operatorname{DG}(\beta)=\left(\mathbb{Z}^{4}\right)^{\star} / \operatorname{im}[B Q]^{\star} \cong \mathbb{Z}$, where the isomorphism can be chosen as $\bar{f}([a, b, c, d])=30 a+20 b+12 c-d$. Therefore, $G \cong \mathbb{T}$, and $\beta^{\vee}:\left(\mathbb{Z}^{3}\right)^{\star} \rightarrow \operatorname{DG}(\beta) \cong \mathbb{Z}$ is given by $f(a, b, c)=30 a+20 b+12 c$. Hence, the homomorphism $G \rightarrow \mathbb{T}^{3}$ describing the $G$-action on $Z_{\Sigma}$ is $t \mapsto\left(t^{30}, t^{20}, t^{12}\right)$. It follows that the corresponding stack is equivalent to a weighted projective stack $\mathcal{P}(30,20,12)$, which has global stabilizer $\mu_{2}$. 


\subsection{Relation with Delzant's construction and labeled poly-} topes. The construction of the quotient stack $\left[\mu^{-1}(\tau) / K\right]$ from a stacky polytope may be viewed as a generalization of Lerman-Tolman's generalization [20] of the Delzant construction, which we review next.

In its original form [20], a labeled polytope is a pair $\left(\Delta,\left\{m_{i}\right\}_{i=1}^{n}\right)$ consisting of a convex simple polytope $\Delta$ in $V^{\star}$, where $V$ is a real vector space, with $n$ facets, $F_{1}, \ldots, F_{n}$, whose relative interiors are labeled with positive integers $m_{1}, \ldots, m_{n}$. The polytope is assumed to be rational with respect to a chosen lattice $N \subset V$. Identifying $V \cong N \otimes \mathbb{R}$, we may denote the primitive inward pointing normals by $\nu_{1} \otimes 1, \ldots, \nu_{n} \otimes 1$. Then defining $\beta: \mathbb{Z}^{n} \rightarrow N$ by the formula $\beta\left(e_{i}\right)=m_{i} \nu_{i}$ gives $(N, \Delta, \beta)$ as a stacky polytope. Furthermore, any stacky polytope with $N$ free can be realized as a labeled polytope. Thus, labeled polytopes are precisely those stacky polytopes for which the $\mathbb{Z}$-module $N$ is a free module.

Given a labeled polytope $\left(\Delta,\left\{m_{i}\right\}_{i=1}^{n}\right)$ in $(N \otimes \mathbb{R})^{\star} \cong\left(\mathbb{R}^{d}\right)^{\star}$, we may proceed with the Delzant construction to obtain a quotient stack $\left[\mu^{-1}(\tau) / K_{\mathrm{D}}\right]$ as a symplectic reduction, where the group $K_{\mathrm{D}} \subset\left(S^{1}\right)^{n}$ acts via the standard linear $\left(S^{1}\right)^{n}$-action on $\mathbb{C}^{n}$. As we shall see below, $K_{D}$ is isomorphic to the group $K=\operatorname{Hom}\left(\operatorname{DG}(\beta), S^{1}\right)$ arising from Sakai's construction. Furthermore, it is straightforward to verify that the isomorphism is compatible with the respective group actions of $K_{D}$ and $K$ on $\mathbb{C}^{n}$, and thus the symplectic quotient stacks $\left[\mu^{-1}(\tau) / K_{D}\right]$ and $\left[\mu^{-1}(\tau) / K\right]$ are equivalent.

The group $K_{\mathrm{D}}$ in the Delzant construction is defined as follows. Let $\beta: \mathbb{Z}^{n} \rightarrow N$ be given by $\beta\left(e_{i}\right)=m_{i} \nu_{i}$, where $m_{i} \nu_{i}$ are the weighted normals to the facets of $\Delta$, and consider the resulting homomorphism $\bar{\beta}:\left(S^{1}\right)^{n} \rightarrow\left(S^{1}\right)^{d}$ induced by $\beta_{\mathbb{R}}=\beta \otimes \mathbb{R}$ (where we have chosen identifications $N \cong \mathbb{Z}^{d}$ and $\left.S^{1} \cong \mathbb{R} / \mathbb{Z}\right)$. Define $K_{\mathrm{D}}=\operatorname{ker} \bar{\beta}$.

To compare the groups $K_{\mathrm{D}}$ and $K$, we note that since $N$ is free, it is easy to verify that $\operatorname{DG}(\beta)=\operatorname{coker} \beta^{\star}$, and hence, we have the short exact sequence

$$
0 \longrightarrow N^{\star} \longrightarrow\left(\mathbb{Z}^{n}\right)^{\star} \longrightarrow \mathrm{DG}(\beta) \longrightarrow 0,
$$

which yields the short exact sequence,

$$
1 \longrightarrow K \longrightarrow \operatorname{Hom}\left(\left(\mathbb{Z}^{n}\right)^{\star}, S^{1}\right) \longrightarrow \operatorname{Hom}\left(N^{\star}, S^{1}\right) \longrightarrow 1 .
$$


Using the natural isomorphism $\operatorname{Hom}\left(M^{\star}, S^{1}\right) \cong M \otimes S^{1}$ (for a free $\mathbb{Z}$-module $M$ ) and identifications

$$
\mathbb{Z}^{n} \otimes S^{1} \cong\left(S^{1}\right)^{n} \quad \text { and } \quad N \otimes S^{1} \cong\left(S^{1}\right)^{d}
$$

resulting from the chosen identification $N \cong \mathbb{Z}^{d}$, we may readily identify $K \cong K_{\mathrm{D}}$.

Example 1.5. Let $N=\mathbb{Z}$. Consider the labeled polytope $\Delta$ in $\mathbb{R} \cong(N \otimes \mathbb{R})^{\star}$ consisting of a line segment with labels $r$ and $s$ at each endpoint (see Figure 2).

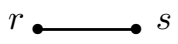

Figure 2. A labeled polytope $\Delta$ in $\mathbb{R}$.

The homomorphism $\beta: \mathbb{Z}^{2} \rightarrow N$ in the corresponding stacky polytope (and stacky fan) is given by the matrix $\beta=\left[\begin{array}{ll}-s & r\end{array}\right]$. Let $g=\operatorname{gcd}(r, s)$. Then $\operatorname{DG}(\beta)=\left(\mathbb{Z}^{2}\right)^{\star} / \operatorname{im} \beta^{\star} \cong \mathbb{Z} \oplus \mathbb{Z} / g \mathbb{Z}$, which may be realized by the isomorphism

$$
\bar{f}([a, b])=\left(\frac{r}{g} a+\frac{s}{g} b,-y a+x b \bmod g\right)
$$

where $x$ and $y$ are integers satisfying $g=x r+y s$. It follows that $G \cong \mathbb{T} \times \mu_{g}$, where $\mu_{g} \subset \mathbb{T}$ is the cyclic group of $g$ th roots of unity. Note that $G$ is connected if and only if $\operatorname{gcd}(r, s)=1$.

Under the above identification, the homomorphism $\beta^{\vee}:\left(\mathbb{Z}^{2}\right)^{\star} \rightarrow$ $\mathrm{DG}(\beta) \cong \mathbb{Z} \oplus \mathbb{Z} / g \mathbb{Z}$ is simply the projection

$$
f(a, b)=\left(\frac{r}{g} a+\frac{s}{g} b,-y a+x b \bmod g \mathbb{Z}\right) .
$$

Therefore, the homomorphism $\mathbb{T} \times \mu_{g} \cong G \rightarrow \mathbb{T}^{2}$ that determines the action on $Z_{\Sigma}=\mathbb{C}^{2} \backslash\{0\}$ is then given by $\left(t, \xi_{g}^{k}\right) \mapsto\left(t^{r / g} \xi_{g}^{-k y}, t^{s / g} \xi_{g}^{k x}\right)$ where $\xi_{g} \in \mathbb{C}$ denotes a primitive $g$ th root of unity.

2. Isotropy and stacky fans. Our goal in this section is to compute the local isotropy group of each point of a toric DM stack, $\mathcal{X}(N, \Sigma, \beta)=\left[Z_{\Sigma} / G\right]$, by computing the subgroup $\operatorname{Stab}(z) \subset G$ that 
fixes a given point $z \in Z_{\Sigma}$. The main result, Theorem 2.2 in subsection 2.1, describes all possible isotropy groups that arise. A discussion of the connected component of $G$ and its role in detecting global quotient stacks appears in subsection 2.2, along with further details in subsection 2.3 for the case of labeled polytopes.

2.1. Isotropy and stacky fans. Recall that $Z_{\Sigma}$ is defined as the complement in $\mathbb{C}^{n}$ of the zero-set of the ideal $J(\Sigma)$, which is described in more detail next.

For $\sigma \in \Sigma$, let $I_{\sigma}=\left\{i: \rho_{i} \subset \sigma\right\}$, and $J_{\sigma}$ its complement. Then,

$$
\begin{aligned}
V(J(\Sigma)) & =\bigcap_{\sigma \in \Sigma}\left\{\left(z_{1}, \ldots, z_{n}\right) \mid \prod_{\rho_{i} \not \subset \sigma} z_{i}=0\right\} \quad \text { implying } \\
Z_{\Sigma}=\mathbb{C}^{n} \backslash V(J(\Sigma)) & =\bigcup_{\sigma \in \Sigma}\left\{\left(z_{1}, \ldots, z_{n}\right) \mid z_{i} \neq 0 \text { whenever } i \in J_{\sigma}\right\} \\
& =\bigcup_{\sigma \in \Sigma}\left\{\left(z_{1}, \ldots, z_{n}\right) \mid I_{z} \subset I_{\sigma}\right\},
\end{aligned}
$$

where $I_{z}=\left\{i \mid z_{i}=0\right\}$.

There is a natural decomposition of $Z_{\Sigma}$ since an inclusion of cones $\sigma^{\prime} \subset \sigma$ yields an inclusion $Z_{\sigma} \subset Z_{\sigma^{\prime}}$, where $Z_{\sigma}:=\left\{\left(z_{1}, \ldots, z_{n}\right): I_{z} \subset\right.$ $\left.I_{\sigma}\right\}$. Furthermore, for any $z=\left(z_{1}, \ldots, z_{n}\right) \in Z_{\sigma}$, there is a cone $\sigma_{z} \subset \sigma$ given by the span of the minimal generators of the rays $\rho_{i}$ with $i \in I_{z}$. This follows from our assumption that $\Sigma$ is simplicial. Since the number of rays $\rho_{i}$ with $i \in I_{\sigma}$ equals the dimension of $\sigma$, any subset of these rays spans a face of $\sigma$ and is thus in the fan $\Sigma$. It follows then that, for every point $z \in Z_{\Sigma}$, we may write $z \in Z_{\sigma_{z}}$ where the cone $\sigma_{z}$ satisfies $\left\{i: z_{i}=0\right\}=I_{\sigma_{z}}$. Moreover, $\sigma_{z}$ is minimal in the sense that $\sigma_{z} \subset \sigma$ for any $\sigma$ such that $z \in Z_{\sigma}$.

For a point $z \in \mathbb{C}^{n}$, the subgroup in $\mathbb{T}^{n}$ fixing $z$ is $\left\{\left(t_{1}, \ldots, t_{n}\right): t_{i}=\right.$ 1 if $\left.z_{i} \neq 0\right\}$, which motivates the following definition. For any subset $I \subset\{1, \ldots, n\}$ and its complement $J$, let

$$
\mathbb{T}^{I}=\left\{\left(t_{1}, \ldots, t_{n}\right): i \in J \text { implies } t_{i}=1\right\} \subseteq \mathbb{T}^{n} .
$$

Note that $\mathbb{T}^{I}$ is the kernel of the map $\mathbb{T}^{n} \rightarrow \mathbb{T}^{|J|}$ given by projection onto the coordinates indicated by $J$ with cardinality $|J|$.

Since $G$ acts on $Z_{\Sigma}$ via the homomorphism $G \rightarrow \mathbb{T}^{n}$ induced by $\beta^{\vee}$, then the isotropy $\operatorname{Stab}(z)$ associated to a point $z \in Z_{\Sigma}$ is given by the 
kernel of the map

$$
G \longrightarrow \mathbb{T}^{n} \stackrel{\pi}{\longrightarrow} \mathbb{T}^{\left|J_{z}\right|},
$$

where $J_{z}$ is the complement of $I_{z}$. At times, it is useful to view the subset $I_{z}$ as $I_{\sigma}$ for the cone $\sigma=\sigma_{z}$ in $\Sigma$ described above, in which case we denote $\operatorname{Stab}(z)$ by $\Gamma_{\sigma}$.

Remark 2.1. Notice that an inclusion of cones $\sigma^{\prime} \subset \sigma$ in $\Sigma$ induces an inclusion $J_{\sigma} \subset J_{\sigma^{\prime}}$ and hence we obtain the following commutative diagram,

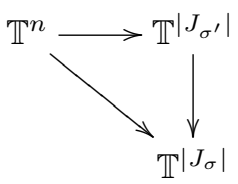

where the vertical map is the natural projection. Therefore, there is a natural inclusion of isotropy groups $\Gamma_{\sigma^{\prime}} \subset \Gamma_{\sigma}$. It follows each such isotropy group is contained in $\Gamma_{\sigma}$ for some maximal cone $\sigma$. In particular, all isotropy groups are trivial if and only if $\Gamma_{\sigma}$ is trivial for maximal cones $\sigma$ in $\Sigma$.

Theorem 2.2. Let $(N, \Sigma, \beta)$ be a stacky fan and $\left[Z_{\Sigma} / G\right]$ its corresponding toric DM stack. For a point $z=\left(z_{1}, \ldots, z_{n}\right)$ in $Z_{\Sigma} \subset \mathbb{C}^{n}$, let $N_{z} \subset N$ denote the submodule generated by $\left\{\beta\left(e_{i}\right) \mid z_{i}=0\right\}$. The isotropy group $\operatorname{Stab}(z)$ is isomorphic to $\operatorname{Hom}\left(\operatorname{Ext}_{\mathbb{Z}}^{1}\left(N / N_{z}, \mathbb{Z}\right), \mathbb{T}\right)$; therefore, $\operatorname{Stab}(z)$ is (non-canonically) isomorphic to the torsion submodule $\operatorname{Tor}\left(N / N_{z}\right)$.

Proof. Let $\sigma$ be a cone in $\Sigma$ with $I_{z}=I_{\sigma}$. We noted above that the stabilizer of $z$ is given by $\Gamma_{\sigma}$, the kernel of the composition (2.1). This composition is realized by applying the functor $\operatorname{Hom}(-, \mathbb{T})$ to the composition $f=\beta^{\vee} \circ \pi^{\star}$,

$$
\left(\mathbb{Z}^{\left|J_{\sigma}\right|}\right)^{\star} \stackrel{\pi^{\star}}{\longrightarrow}\left(\mathbb{Z}^{n}\right)^{\star} \stackrel{\beta^{\vee}}{\longrightarrow} \mathrm{DG}(\beta)
$$

where $\pi^{\star}$ is inclusive of the relevant factors. Moreover, since $\mathbb{T}$ is injective as a $\mathbb{Z}$-module, the kernel of equation $(2.1)$ is $\operatorname{Hom}(\operatorname{coker} f, \mathbb{T})$. As we shall see next, coker $f \cong \operatorname{Ext}_{\mathbb{Z}}^{1}\left(N / N_{\sigma}, \mathbb{Z}\right)$, where $N_{\sigma}=N_{z} \subset N$ denotes the subgroup generated by $\left\{\beta\left(e_{i}\right) \mid i \in I_{\sigma}\right\}$. 
Let $\beta_{\sigma}: \mathbb{Z}^{I_{\sigma}} \rightarrow N_{\sigma}$ denote the restriction of $\beta$ to $\mathbb{Z}^{I_{\sigma}}$ together with its codomain. We claim that $\beta_{\sigma}: \mathbb{Z}^{I_{\sigma}} \rightarrow N_{\sigma}$ is an isomorphism, and hence $N_{\sigma}$ is free. That $\Sigma$ is simplicial means that the $\left\{\beta\left(e_{i}\right) \otimes 1\right\}_{i \in I_{\sigma}}$ are linearly independent in $N \otimes \mathbb{R}$. Therefore, $\operatorname{rank} N_{\sigma}=\left|I_{\sigma}\right|$. Since $\beta_{\sigma}$ is a surjective homomorphism of modules of the same rank, $\beta_{\sigma} \otimes \mathbb{R}$ is an isomorphism of vector spaces. But, since the domain $\mathbb{Z}^{I_{\sigma}}$ of $\beta_{\sigma}$ is free, $\beta_{\sigma}$ must be injective as well. This verifies the claim.

In particular, this implies that $\operatorname{DG}\left(\beta_{\sigma}\right)$ is trivial. Any lift $B_{\sigma}$ of $\beta_{\sigma}$ is an isomorphism and $N_{\sigma}$ has no torsion, so $\mathrm{DG}\left(\beta_{\sigma}\right)=\operatorname{coker}\left[B_{\sigma}\right]^{\star}=$ $\operatorname{coker} \beta_{\sigma}^{\star}$.

Consider the following diagram, whose rows are exact.

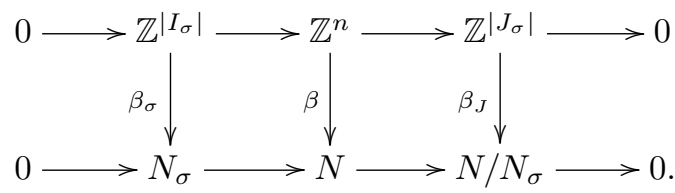

By [5, Lemma 2.3], we get the following commutative diagram with exact rows, noting that $\mathrm{DG}\left(\beta_{\sigma}\right)$ is trivial.

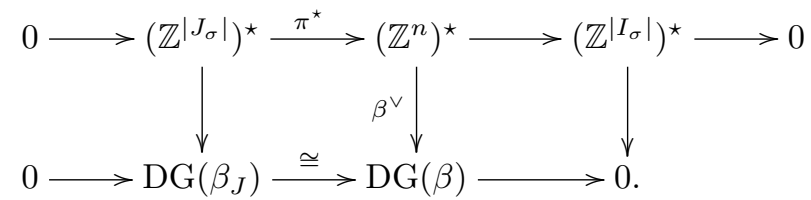

We identify $f=\beta^{\vee} \circ \pi^{\star}$ with the left vertical arrow.

Applying the exact sequence from $[\mathbf{5},(2.0 .3)]$ to $\beta_{J}: \mathbb{Z}^{\left|J_{\sigma}\right|} \rightarrow N / N_{\sigma}$, we get

$$
\left(N / N_{\sigma}\right)^{\star} \longrightarrow\left(\mathbb{Z}^{\left|J_{\sigma}\right|}\right)^{\star} \longrightarrow \mathrm{DG}\left(\beta_{J}\right) \longrightarrow \operatorname{Ext}_{\mathbb{Z}}^{1}\left(N / N_{\sigma}, \mathbb{Z}\right) \longrightarrow 0,
$$

whence coker $(f) \cong \operatorname{Ext}_{\mathbb{Z}}^{1}\left(N / N_{\sigma}, \mathbb{Z}\right)$, as required. Since $\operatorname{Ext}_{\mathbb{Z}}^{1}\left(N / N_{\sigma}, \mathbb{Z}\right)$ is (non-canonically) isomorphic to (the finite abelian group) $\operatorname{Tor}\left(N / N_{\sigma}\right)$, it follows that $\operatorname{Hom}\left(\operatorname{Ext}_{\mathbb{Z}}^{1}\left(N / N_{\sigma}, \mathbb{Z}\right), \mathbb{T}\right)$ is isomorphic to $\operatorname{Tor}\left(N / N_{\sigma}\right)$.

Example 2.3. Consider the toric DM stack from Example 1.3. We compute the isotropy for the points $z=(0, a, 0)$ and $w=(0, a, b)$ of the form in $Z_{\Sigma}$ with $a, b \neq 0$. Since $I_{z}=\{1,3\}$, then $N_{z} \subset N$ is 
the subgroup generated by $(0,5)$ and $(-2,-2)$. Therefore, $N / N_{z} \cong$ $\mathbb{Z} / 5 \mathbb{Z} \oplus \mathbb{Z} / 2 \mathbb{Z}$ and $\operatorname{Stab}(z) \cong \operatorname{Tor}\left(N / N_{z}\right) \cong \mu_{10}$.

Since $I_{w}=\{1\}$, then $N_{w}$ is the subgroup generated by $(-2,-2)$, and $N / N_{w} \cong \mathbb{Z} \oplus \mathbb{Z} / 2 \mathbb{Z}$. Therefore, $\operatorname{Stab}(w) \cong \operatorname{Tor}\left(N / N_{w}\right) \cong \mu_{2}$. Note that the isotropy for $w$ can simply be read off from the corresponding facet label (see Figure 1) in this case. For higher-dimensional cones, a more detailed analysis is required, e.g., see subsection 4.3.

The proof of Theorem 2.2 does not explicitly show how $\operatorname{Tor}\left(N / N_{z}\right)$ may be viewed as a subgroup of $G$. Our next goal is Proposition 2.4 which gives an explicit identification of the stabilizer group and $\operatorname{Tor}\left(N / N_{z}\right)$. To accomplish this, we first construct a map $\operatorname{Tor}\left(N / N_{z}\right) \rightarrow$ $G$ (2.2). (See Proposition 2.15 for a more direct approach in the case that $N$ is free.) For any cone $\sigma$ in $\Sigma$, let $N_{\sigma}$ be the subgroup generated by $\left\{\beta\left(e_{i}\right) \mid i \in I_{\sigma}\right\}$. We define a map

$$
\gamma_{\sigma}: \operatorname{Tor}\left(N / N_{\sigma}\right) \longrightarrow \operatorname{Hom}(\mathrm{DG}(\beta), \mathbb{T})
$$

that depends on a choice of resolution

$$
0 \longrightarrow \mathbb{Z}^{\ell} \stackrel{R}{\longrightarrow} \mathbb{Z}^{d-I+\ell} \stackrel{q}{\longrightarrow} N / N_{\sigma} \longrightarrow 0,
$$

and a lift $\widetilde{q}: \mathbb{Z}^{d-I+\ell} \rightarrow N$ (cf., [27, Lemma 2.2.8]), where $I=\left|I_{\sigma}\right|$. Given $x \in \operatorname{Tor}\left(N / N_{\sigma}\right)$, choose a representative $a$ in $\mathbb{Z}^{d-I+\ell}$. We shall define a homomorphism $\phi_{a}\left(\mathbb{Z}^{n+\ell}\right)^{*} \rightarrow \mathbb{C}$ that descends to a homomorphism $\bar{\phi}_{a}: \operatorname{DG}(\beta) \rightarrow \mathbb{C} / \mathbb{Z} \cong \mathbb{T}$ and set $\gamma_{\sigma}(x)=\bar{\phi}_{a}$.

Set $J=n-I$, and write elements of $\left(\mathbb{Z}^{n+\ell}\right)^{\star} \cong\left(\mathbb{Z}^{I}\right)^{\star} \oplus\left(\mathbb{Z}^{J}\right)^{\star} \oplus\left(\mathbb{Z}^{\ell}\right)^{\star}$ as triples $\left(u_{I}, u_{J}, v\right)$. Define $\phi_{a}\left(u_{I}, u_{J}, v\right)=u_{I}(b)+v(c)$, where $b$ is the unique element in $\mathbb{Z}^{I} \otimes \mathbb{C}$ satisfying $\left(\beta_{\sigma}\right)_{\mathbb{C}}(b)=\widetilde{q}_{\mathbb{C}}(a)$ and $c$ is the unique element in $\mathbb{Z}^{\ell} \otimes \mathbb{C}$ satisfying $R_{\mathbb{C}}(c)=a$.

To verify that $\phi_{a}$ descends to a homomorphism in $\operatorname{Hom}(\operatorname{DG}(\beta), \mathbb{T})$, we choose the resolution

$$
0 \longrightarrow \mathbb{Z}^{\ell} \stackrel{Q}{\longrightarrow} \mathbb{Z}^{d+\ell} \longrightarrow N \longrightarrow 0
$$

with $Q v=\left(-\beta_{\sigma}^{-1} \widetilde{q} R v, R v\right)$, and a lift $B: \mathbb{Z}^{n} \rightarrow \mathbb{Z}^{I} \oplus \mathbb{Z}^{d-I+\ell}$ satisfying $B(b, 0)=a-Q(c)$, where $(b, 0) \in \mathbb{Z}^{n} \cong \mathbb{Z}^{I} \oplus \mathbb{Z}^{J}$. (Such a lift $B$ can be obtained by first choosing a lift $B_{J}: \mathbb{Z}^{J} \rightarrow \mathbb{Z}^{d-I+\ell}$ and setting $B\left(z_{I}, z_{J}\right)=\left(z_{I}+\beta_{\sigma}^{-1}\left(\beta\left(0, z_{J}\right)-\widetilde{q} B_{J} z_{J}\right), B_{J} z_{J}\right)$.) $\quad$ To see that $\phi_{a} \circ[B Q]^{\star}$ has image in $\mathbb{Z} \subset \mathbb{C}$, note that, for $\left[\begin{array}{lll}u_{I} & w & v\end{array}\right]$ in 


$$
\begin{aligned}
\left(\mathbb{Z}^{d+\ell}\right)^{\star} \cong\left(\mathbb{Z}^{I}\right)^{\star} \oplus\left(\mathbb{Z}^{d-I}\right)^{\star} \oplus\left(\mathbb{Z}^{\ell}\right)^{\star} & \\
\phi_{a}[B Q]^{\star}\left[\begin{array}{lll}
u_{I} & w & v
\end{array}\right] & =\left[\begin{array}{lll}
u_{I} & w & v
\end{array}\right][B Q]\left[\begin{array}{l}
b \\
0 \\
c
\end{array}\right] \\
& =\left[\begin{array}{lll}
u_{I} & w & v
\end{array}\right](B(b, 0)+Q(c)) \\
& =v(a) \in \mathbb{Z} .
\end{aligned}
$$

Notice that a different choice of representative $a^{\prime}=a+R w, w \in \mathbb{Z}^{\ell}$, for $x$ leads to a homomorphism $\phi_{a^{\prime}}$ that differs from $\phi_{a}$ by an integervalued function $\phi_{R w}$; therefore, $\gamma_{\sigma}$ in equation (2.2) is well defined.

Proposition 2.4. The homomorphism $\gamma_{\sigma}$ in equation (2.2) induces an isomorphism $\operatorname{Tor}\left(N / N_{\sigma}\right) \cong \Gamma_{\sigma}$.

Proof. We verify that $\gamma_{\sigma}$ induces an isomorphism $\operatorname{Tor}\left(N / N_{\sigma}\right) \cong \Gamma_{\sigma}$ by checking that $\gamma_{\sigma}$ is injective and that the composition

$$
\operatorname{Tor}\left(N / N_{\sigma}\right) \stackrel{\gamma_{\sigma}}{\longrightarrow} G \longrightarrow \mathbb{T}^{n} \longrightarrow \mathbb{T}^{J}
$$

is trivial. If $\gamma_{\sigma}(x)=0$, then $\phi_{a}$ is integer-valued, whence the corresponding element $c \in \mathbb{C}^{\ell}$ must actually lie in $\mathbb{Z}^{\ell}$ and thus $x=q(a)=0$. Therefore, $\gamma_{\sigma}$ is injective. Finally, the last two maps in the above composition are obtained by pulling back a homomorphism $\operatorname{DG}(\beta) \rightarrow \mathbb{T}$ along the quotient map $\left(\mathbb{Z}^{I+J+\ell}\right)^{\star} \rightarrow \operatorname{DG}(\beta)$ and the composite $\left(\mathbb{Z}^{J}\right)^{\star} \rightarrow\left(\mathbb{Z}^{I+J}\right)^{\star} \rightarrow\left(\mathbb{Z}^{I+J+\ell}\right)^{\star}$ of inclusions. By definition of $\gamma_{\sigma}$, this pullback is trivial, since $\phi_{a}$ is trivial on elements of the form $\left[\begin{array}{lll}0 & u_{J} & 0\end{array}\right]$.

Remark 2.5. In practice, the isotropy groups in Theorem 2.2 may be computed using the Smith normal form of a matrix, as we outline next. As in the proof of the theorem, the isotropy group $\Gamma_{\sigma}$ is isomorphic to the torsion subgroup of the cokernel of the composition $\mathbb{Z}^{\left|I_{\sigma}\right|} \rightarrow \mathbb{Z}^{n} \stackrel{\beta}{\rightarrow} N$. As in the discussion following Definition 1.1, choose a free resolution

$$
0 \longrightarrow \mathbb{Z}^{\ell} \stackrel{Q}{\longrightarrow} \mathbb{Z}^{d+\ell} \longrightarrow N
$$

and a lift $B: \mathbb{Z}^{n} \rightarrow \mathbb{Z}^{d+\ell}$ of $\beta$, and let $B_{\sigma}$ denote the restriction of $B$ 
to $\mathbb{Z}^{\left|I_{\sigma}\right|}$. Then, the commutative diagram

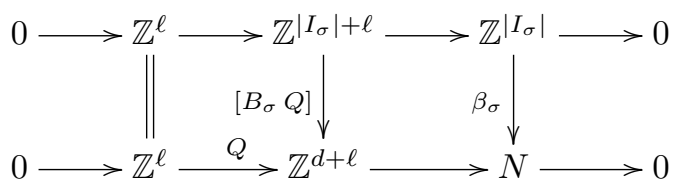

of short exact sequences shows that $\operatorname{coker}\left[B_{\sigma} Q\right]$ and coker $\beta_{\sigma}$ are isomorphic. Thus, it suffices to compute the torsion submodule of the cokernel of the matrix $\left[B_{\sigma} Q\right]$. The Smith normal form of $\left[\begin{array}{ll}B_{\sigma} & Q\end{array}\right]$ will be a $(d+\ell) \times\left(\left|I_{\sigma}\right|+\ell\right)$ matrix with non-zero entries $a_{1}, a_{2}, \ldots, a_{\min \left(d+\ell,\left|I_{\sigma}\right|+\ell\right)}$ appearing on the diagonal, satisfying the divisibility relations $a_{j} \mid a_{j+1}$. The entries $a_{j} \neq 1$ give the orders of the cyclic subgroups appearing in the invariant factor decomposition of $\Gamma_{\sigma}$.

Example 2.6. To illustrate Remark 2.5, we consider the following example. Let $N=\mathbb{Z}^{2} \oplus \mathbb{Z} / 2 \mathbb{Z}$, and let $\Sigma$ be the fan in Figure 3, with ray generators $(1,0),(0,1),(0,-1)$ and $(-1,-2)$. Set $\beta: \mathbb{Z}^{4} \rightarrow N$ to be

$$
\beta(x, y, z, w)=(-2 x+3 z,-4 x+6 y-2 w, x+y+z+w \bmod 2) .
$$

Fix the resolution $0 \rightarrow \mathbb{Z} \stackrel{Q}{\rightarrow} \mathbb{Z}^{3} \rightarrow N \rightarrow 0$ with $Q=\left[\begin{array}{lll}0 & 0 & 2\end{array}\right]^{T}$, and choose $B: \mathbb{Z}^{4} \rightarrow \mathbb{Z}^{3}$ so that

$$
\left[\begin{array}{ll}
B & Q
\end{array}\right]=\left[\begin{array}{ccccc}
-2 & 0 & 3 & 0 & 0 \\
-4 & 6 & 0 & -2 & 0 \\
1 & 1 & 1 & 1 & 2
\end{array}\right]
$$

Let $\sigma$ be the cone in $\Sigma$ generated by $\rho_{1}$ and $\rho_{2}$. Then since

$$
\left[\begin{array}{ll}
B_{\sigma} & Q
\end{array}\right]=\left[\begin{array}{ccc}
-2 & 0 & 0 \\
-4 & 6 & 0 \\
1 & 1 & 2
\end{array}\right] \text { has Smith normal form }\left[\begin{array}{ccc}
1 & 0 & 0 \\
0 & 2 & 0 \\
0 & 0 & 12
\end{array}\right]
$$

we see that the isotropy group $\Gamma_{\sigma} \cong \mu_{2} \times \mu_{12}$.

Notice that, if $\sigma$ is maximal, then $N_{\sigma}$ has the same rank as $N$. The following corollary is immediate. (Compare with [5, Proposition 4.3].) 

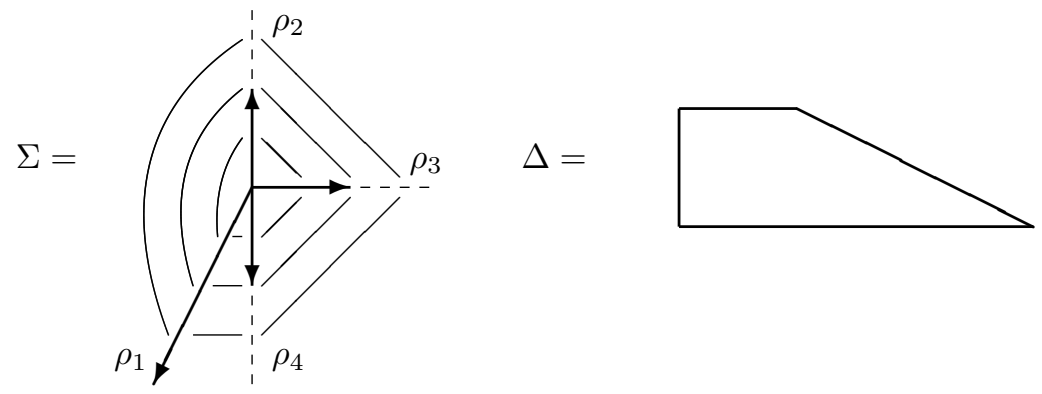

Figure 3. A polytope $\Delta$ in $\mathbb{R}^{2} \cong(N \otimes \mathbb{R})^{\star}$ and its dual fan $\Sigma=\Sigma(\Delta)$, from Example 2.6.

Corollary 2.7. Let $(N, \Sigma, \beta)$ be a stacky fan, and $\left[Z_{\Sigma} / G\right]$ its corresponding toric $\mathrm{DM}$ stack. If $z=\left(z_{1}, \ldots, z_{n}\right) \in Z_{\Sigma}$ has $d=\operatorname{rank} N$ vanishing coordinates, then $\sigma=\sigma_{z}$ is maximal and $\operatorname{Stab}(z) \cong N / N_{z}$.

2.2. Global quotients among toric DM stacks. It is of interest to determine when a given stack $\mathcal{X}$ is a global quotient in the sense of stacks. The goal of this subsection is to characterize the global quotients among toric DM stacks in terms of the combinatorics of the stacky fan and to give an explicit description of the quotient stack $\left[Z_{\Sigma} / G_{0}\right]$, which [14] shows is the universal cover (in the sense of stacks) of a toric DM stack $\left[Z_{\Sigma} / G\right]$. Similar results are also obtained in [14, Proposition 5.5, Corollary 5.7].

Let $G_{0} \subset G$ denote the connected component of the identity element. By construction, the short exact sequence $0 \rightarrow \operatorname{Tor}(\operatorname{DG}(\beta)) \rightarrow$ $\mathrm{DG}(\beta) \rightarrow \mathrm{DG}(\beta) / \operatorname{Tor}(\mathrm{DG}(\beta)) \rightarrow 0$ dualizes to give the short exact sequence $1 \rightarrow G_{0} \rightarrow G \rightarrow G / G_{0} \rightarrow 1$. Below, we identify $G_{0}$ and the quotient $G / G_{0}$ in terms of stacky fan data.

Lemma 2.8. Let $(\Sigma, N, \beta)$ be a stacky fan, and $G=\operatorname{Hom}(\operatorname{DG}(\beta), \mathbb{T})$. If $G_{0} \subset G$ denotes the connected component of the identity element, then $G / G_{0} \cong \operatorname{coker} \beta$. In particular, $G$ is connected if and only if $\beta$ is surjective. 
Proof. As $G=\operatorname{Hom}(\operatorname{DG}(\beta), \mathbb{T})$, the group of connected components $G / G_{0}$ is $\operatorname{Hom}(\operatorname{Tor}(\operatorname{DG}(\beta)), \mathbb{T})$, where $\operatorname{Tor}(\operatorname{DG}(\beta))$ denotes the torsion submodule of $\mathrm{DG}(\beta)$. This torsion submodule may be identified by computing $\operatorname{Ext}_{\mathbb{Z}}^{1}(\operatorname{DG}(\beta), \mathbb{Z})$, which we show next is isomorphic to coker $\beta$.

Note that coker $\beta \cong \operatorname{coker}\left[\begin{array}{ll}B & Q\end{array}\right]$, where $\left[\begin{array}{ll}B & Q\end{array}\right]: \mathbb{Z}^{n+\ell} \rightarrow \mathbb{Z}^{d+\ell}$ is the homomorphism described in section 1 . This can be seen by verifying that the surjective composition $\mathbb{Z}^{d+\ell} \rightarrow N \rightarrow \operatorname{coker} \beta$ has kernel im $\left[\begin{array}{ll}B & Q\end{array}\right]$. Therefore, it suffices to show that $\operatorname{Ext}_{\mathbb{Z}}^{1}(\operatorname{DG}(\beta), \mathbb{Z}) \cong$ coker $\left[\begin{array}{ll}B & Q\end{array}\right]$.

To this end, consider the free resolution that defines $\operatorname{DG}(\beta)$ :

$$
0 \longrightarrow\left(\mathbb{Z}^{d+\ell}\right)^{\star} \stackrel{[B Q]^{\star}}{\longrightarrow}\left(\mathbb{Z}^{n+\ell}\right)^{\star} \longrightarrow \mathrm{DG}(\beta) \longrightarrow 0
$$

(Note that since coker $\left[\begin{array}{ll}B & Q\end{array}\right] \cong \operatorname{coker} \beta$ is assumed to be finite, then $\left[\begin{array}{ll}B & Q\end{array}\right]^{\star}$ is injective.) Applying $\operatorname{Hom}(-, \mathbb{Z})$ and taking homology shows that $\operatorname{Ext}_{\mathbb{Z}}^{1}(\operatorname{DG}(\beta), \mathbb{Z}) \cong \operatorname{coker}[B \quad Q]$, as required.

Remark 2.9. By [14, Corollary 3.5], Lemma 2.8 shows that the (stacky) fundamental group of $\mathcal{X}(N, \Sigma, \beta)$ is therefore isomorphic to coker $\beta$, cf., [10, Section 3.2].

Remark 2.10. As in the proof of Lemma 2.8, $\operatorname{coker} \beta \cong \operatorname{coker}\left[\begin{array}{ll}B \quad & Q\end{array}\right]$ and thus the invariant factor decomposition of $G / G_{0}$ may be determined immediately from the Smith normal form of the matrix $\left[\begin{array}{ll}B & Q\end{array}\right]$. For example, the reader may verify that $G / G_{0} \cong \mathbb{Z} / 2 \mathbb{Z}$ in Example 2.6.

Given a stacky fan $(N, \Sigma, \beta)$, we may model the quotient stack $\left[Z_{\Sigma} / G_{0}\right]$ as a toric DM stack of a related stacky fan $\left(N_{0}, \Sigma_{0}, \beta_{0}\right)$, defined as follows. Consider the submodule $N_{0}=\operatorname{im}(\beta) \subset N$, and let $\beta_{0}: \mathbb{Z}^{n} \rightarrow N_{0}$ be given by $\beta$ with its restricted codomain. Finally, we let $\Sigma_{0}$ be the fan in $N_{0} \otimes \mathbb{R}$ corresponding to $\Sigma$ defined by the natural isomorphism $N_{0} \otimes \mathbb{R} \cong N \otimes \mathbb{R}$ induced by the inclusion $N_{0} \subset N$ (where we have used the fact that $\beta$ has finite cokernel).

Proposition 2.11. Let $(N, \Sigma, \beta)$ be a stacky fan and $\mathcal{X}(N, \Sigma, \beta)=$ $\left[Z_{\Sigma} / G\right]$ its corresponding toric DM stack. The triple $\left(N_{0}, \Sigma_{0}, \beta_{0}\right)$ 
defined as above is a stacky fan whose corresponding toric DM stack is $\mathcal{X}\left(N_{0}, \Sigma_{0}, \beta_{0}\right)=\left[Z_{\Sigma} / G_{0}\right]$.

Proof. It is straightforward to verify that $\left(N_{0}, \Sigma_{0}, \beta_{0}\right)$ defines a stacky fan. Since $\Sigma_{0}$ and $\Sigma$ contain the same combinatorial information, $J(\Sigma)=J\left(\Sigma_{0}\right)$, and thus $Z_{\Sigma_{0}}=Z_{\Sigma}$.

It remains to verify that the group action on $Z_{\Sigma}$ determined by the stacky fan $\left(N_{0}, \Sigma_{0}, \beta_{0}\right)$ is the same as that obtained by the restriction of the action of $G$ to the connected component of the identity $G_{0}$ on $Z_{\Sigma}$.

To see this, we apply [5, Lemma 2.3] to the following diagram of short exact sequences.

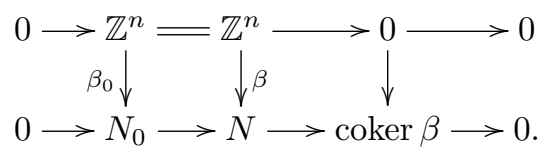

Since $\operatorname{DG}(\{0\} \rightarrow \operatorname{coker}(\beta))$ can be naturally identified with coker $(\beta)$, we obtain the diagram below with exact rows.

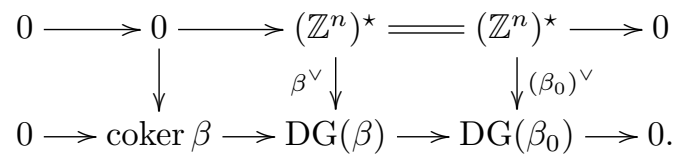

This shows that $\operatorname{DG}\left(\beta_{0}\right)$ and $\operatorname{DG}(\beta)$ have the same rank and thus $\operatorname{Hom}\left(\operatorname{DG}\left(\beta_{0}\right), \mathbb{T}\right)$ and $G$ have the same dimension. To show that $\operatorname{Hom}\left(\operatorname{DG}\left(\beta_{0}\right), \mathbb{T}\right)$ is connected, it suffices to verify that $\operatorname{DG}\left(\beta_{0}\right)$ is torsion free, which follows from Lemma 2.8.

Thus, $\operatorname{Hom}\left(\operatorname{DG}\left(\beta_{0}\right), \mathbb{T}\right)=G_{0}$, the connected component of the identity in $G$. Lastly, note that the $G_{0}$ action on $\mathbb{Z}_{\Sigma}$ is induced by the composition $\left(\mathbb{Z}^{n}\right)^{\star} \stackrel{\beta^{\vee}}{\rightarrow} \mathrm{DG}(\beta) \rightarrow \mathrm{DG}\left(\beta_{0}\right)$ so that $G_{0}$ acts via its inclusion into $G$, as desired.

The above discussion yields a combinatorial condition characterizing global quotients among toric DM stacks.

Corollary 2.12. Let $(N, \Sigma, \beta)$ be a stacky fan, and let $N_{0}=\operatorname{im}(\beta)$. 
(i) $N_{\sigma}=N_{0}$ for all maximal cones $\sigma \in \Sigma$ if and only if $G_{0}$ acts freely on $Z_{\Sigma}$.

(ii) $N_{\sigma}=N$ for all maximal cones $\sigma \in \Sigma$ if and only if $G$ is connected and acts freely on $Z_{\Sigma}$.

As mentioned above, Corollary 2.12 (i) gives a criterion for detecting global quotients among toric DM stacks. In particular, it shows that if $N_{\sigma}=N_{0}$ for all maximal cones, the toric DM stack $\mathcal{X}\left(N_{0}, \Sigma_{0}, \beta_{0}\right)=$ $\left[Z_{\Sigma} / G_{0}\right]$ is in fact a smooth manifold. As shown in [14], this exhibits the toric DM stack $\mathcal{X}(N, \Sigma, \beta)$ as a global quotient in this case-more precisely, when $N_{\sigma}=N_{0}$ for all maximal cones $\sigma \in \Sigma$, there is a natural equivalence of stacks $\mathcal{X}(N, \Sigma, \beta) \cong\left[\left(Z_{\Sigma} / G_{0}\right) / \Lambda\right]$ where $\Lambda=G / G_{0}$.

Example 2.13. Consider the toric DM stack $\left[Z_{\Sigma} / G\right]$ from Example 1.5, the labeled line segment with labels $r$ and $s$. Since $N_{0}=g \mathbb{Z}$ where $g=\operatorname{gcd}(r, s),\left[Z_{\Sigma} / G\right]$ is a global quotient if and only if $r=g=s$. In that case, the $G \cong \mathbb{T} \times \mu_{r}$-action on $Z_{\Sigma}$ is induced by the homomorphism $G \rightarrow \mathbb{T}^{2},\left(t, \xi_{r}^{k}\right) \mapsto\left(t, t \xi_{r}^{k}\right)$. Therefore, $Z_{\Sigma} / G_{0}=\mathbb{P}^{1}$ and the residual $\Lambda=G / G_{0} \cong \mu_{r}$-action may be written in homogeneous coordinates as $\xi_{r}^{k} \cdot\left[z_{0}: z_{1}\right]=\left[z_{0}: \xi_{r}^{k} z_{1}\right]$ and $\left[Z_{\Sigma} / G\right] \cong\left[\mathbb{P}^{1} / \mu_{r}\right]$.

Remark 2.14. Analogous to the map constructed in Proposition 2.4 modeling the inclusion of the isotropy groups, we may also model the quotient map $G \rightarrow G / G_{0}$, cf., Lemma 2.8, more concretely as in the discussion that follows. See Proposition 2.17 for a more direct approach in the case that $N$ is free.

Recall that the quotient $G \rightarrow G / G_{0}$ is the map $\operatorname{Hom}(\operatorname{DG}(\beta), \mathbb{T}) \rightarrow$ $\operatorname{Hom}(\operatorname{Tor}(\mathrm{DG}(\beta)), \mathbb{T})$, induced by the inclusion of the torsion submodule $\operatorname{Tor}(\mathrm{DG}(\beta)) \hookrightarrow \mathrm{DG}(\beta)$. We shall describe an explicit isomorphism

$$
\operatorname{Hom}(\operatorname{Tor}(\mathrm{DG}(\beta)), \mathbb{T}) \stackrel{\cong}{\longrightarrow} \operatorname{coker}[B Q]
$$

Consider the diagram of short exact sequences,

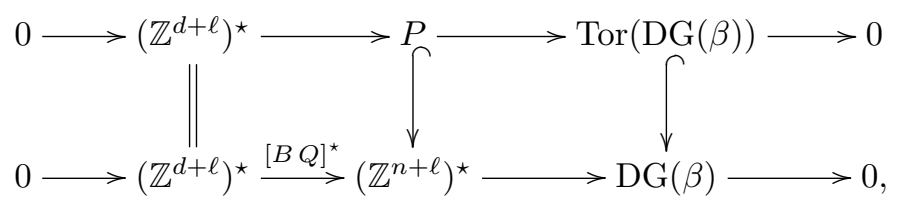


obtained by restriction (pullback) to $\operatorname{Tor}(\operatorname{DG}(\beta))$. Given a homomorphism $\varphi: \operatorname{Tor}(\operatorname{DG}(\beta)) \rightarrow \mathbb{T}=\mathbb{C} / \mathbb{Z}$, choose a homomorphism $\widehat{\varphi}: P \rightarrow \mathbb{C}$ covering $\varphi$. The restriction $\left.\widehat{\varphi}\right|_{\left(\mathbb{Z}^{d+\ell}\right)^{\star}}$ is integer-valued, and hence defines a vector $v_{\hat{\varphi}} \in \mathbb{Z}^{d+\ell}$ by duality (i.e., $\left.\hat{\varphi}\right|_{\left(\mathbb{Z}^{d+\ell}\right)^{\star}}(u)=u v_{\hat{\varphi}}$ for all row vectors $\left.u \in\left(\mathbb{Z}^{d+\ell}\right)^{\star}\right)$.

Any two covers $\widehat{\varphi}_{1}, \widehat{\varphi}_{2}$ of $\varphi$ differ by a homomorphism $\alpha$ : $P \rightarrow \mathbb{Z}$, which by restriction to $\left(\mathbb{Z}^{d+\ell}\right)^{\star}$ defines a vector $v_{\alpha} \in$ $\mathbb{Z}^{d+\ell}$ that is the difference between $v_{\hat{\varphi}_{1}}$ and $v_{\hat{\varphi}_{2}}$. We check that $v_{\alpha}$ is in the image of $\left[\begin{array}{ll}B & Q\end{array}\right]: \mathbb{Z}^{n+\ell} \rightarrow \mathbb{Z}^{d+\ell}$, and hence the correspondence $\varphi \mapsto v_{\hat{\varphi}}$ descends to a well-defined homomorphism $\operatorname{Hom}(\operatorname{Tor}(\operatorname{DG}(\beta)), \mathbb{T}) \rightarrow \operatorname{coker}\left[\begin{array}{ll}B & Q\end{array}\right]$. Applying the Snake lemma to the diagram of short exact sequences above shows that the quotient $\left(\mathbb{Z}^{n+\ell}\right)^{\star} / P \cong \mathrm{DG}(\beta) / \operatorname{Tor}(\mathrm{DG}(\beta))$ is free; therefore, $\alpha: P \rightarrow \mathbb{Z}$ may be extended to a homomorphism $\widetilde{\alpha}:\left(\mathbb{Z}^{n+\ell}\right)^{\star} \rightarrow \mathbb{Z}$, which by duality defines a vector $w_{\tilde{\alpha}}$ in $\mathbb{Z}^{n+\ell}$. Then for any row vector $u \in\left(\mathbb{Z}^{d+\ell}\right)^{\star}$, we have

$$
u v_{\alpha}=\left.\alpha\right|_{\left(\mathbb{Z}^{d+\ell}\right)^{\star}}(u)=\widetilde{\alpha}\left(u\left[\begin{array}{ll}
B & Q
\end{array}\right]\right)=u\left[\begin{array}{ll}
B & Q
\end{array}\right] w_{\tilde{\alpha}},
$$

and hence, $v_{\alpha}=[B Q] w_{\tilde{\alpha}}$, as required.

A similar argument shows that $\operatorname{Hom}(\operatorname{Tor}(\operatorname{DG}(\beta)), \mathbb{T}) \rightarrow \operatorname{coker}\left[\begin{array}{ll}B & Q\end{array}\right]$ is injective, which implies it is also surjective since these groups are known to be abstractly isomorphic finite groups.

2.3. Isotropy and labeled polytopes. Let $N$ be a free $\mathbb{Z}$-module of rank $d$. Viewing stacky polytopes as labeled polytopes, as in subsection 1.2, we now give a more direct description of the maps modeling the inclusion of isotropy groups $\operatorname{Stab}(z) \hookrightarrow G$ (see, Theorem 2.2 and Proposition 2.4), and the quotient map $G \rightarrow G / G_{0}$ (see Lemma 2.8 and the discussion following Remark 2.14).

Let $\left(\Delta,\left\{m_{i}\right\}_{i=1}^{n}\right)$ in $(N \otimes \mathbb{R})^{\star} \cong\left(\mathbb{R}^{d}\right)^{\star}$ be a labeled polytope. Let $\beta: \mathbb{Z}^{n} \rightarrow N$ be given by $\beta\left(e_{i}\right)=m_{i} \nu_{i}$, where $m_{i} \nu_{i}$ are the weighted normals to the facets of $\Delta$, and consider the resulting homomorphism $\bar{\beta}:\left(S^{1}\right)^{n} \rightarrow\left(S^{1}\right)^{d}$ with kernel $K_{\mathrm{D}}=\operatorname{ker} \bar{\beta}$. Let $\exp : \mathbb{Z}^{n} \otimes \mathbb{R} \rightarrow\left(S^{1}\right)^{n}$ denote the exponential map.

We begin with an explicit description of the stabilizers of Theorem 2.2. 
Proposition 2.15. Let $\left(\Delta,\left\{m_{i}\right\}_{i=1}^{n}\right)$ be a labeled polytope in $(N \otimes \mathbb{R})^{\star}$ with primitive inward pointing facet normals $\nu_{1} \otimes 1, \ldots, \nu_{n} \otimes 1$ and $\left[\mu^{-1}(\tau) / K_{D}\right]$ its corresponding toric DM stack. For $z=\left(z_{1}, \ldots, z_{n}\right)$ in $\mu^{-1}(\tau) \subset \mathbb{C}^{n}$, let $N_{z} \subset N$ denote the submodule generated by $\left\{m_{i} \nu_{i} \mid z_{i}=0\right\}$. Then the canonical map

$$
\operatorname{Tor}\left(N / N_{z}\right) \longrightarrow K_{D}, \quad x+N_{z} \longmapsto \exp \left(y \otimes \frac{1}{m}\right),
$$

is an isomorphism onto its image $\operatorname{Stab}(z)$, where $y$ is the unique element in $\operatorname{span}\left\{e_{i} \mid z_{i}=0\right\} \subset \mathbb{Z}^{n}$ satisfying $\beta(y)=m x$ for some smallest positive integer $m$.

Proof. Let $\sigma$ be a cone in the normal fan $\Sigma(\Delta)$ with $I_{\sigma}=I_{z}$. Analogous to subsection 2.1, we see that $\operatorname{Stab}(z)$ is given by the kernel $\Gamma_{\sigma}$ of the composition (cf., (2.1))

$$
K_{\mathrm{D}} \longrightarrow\left(S^{1}\right)^{n} \longrightarrow\left(S^{1}\right)^{\left|J_{\sigma}\right|} .
$$

We exhibit an isomorphism $\psi: \operatorname{Tor}\left(N / N_{\sigma}\right) \rightarrow \Gamma_{\sigma}$, for any cone $\sigma$ of the normal fan $\Sigma(\Delta)$.

Define $\psi: \operatorname{Tor}\left(N / N_{\sigma}\right) \rightarrow \Gamma_{\sigma}$ as follows. Given $x+N_{\sigma}$ of order $m$, we may find a unique (see the claim in Theorem 2.2) $y \in \mathbb{Z}^{I_{\sigma}} \subset \mathbb{Z}^{n}$ with $\beta(y)=m x$. Consider $y \otimes 1 / m \in \mathbb{Z}^{n} \otimes \mathbb{R}$. Since $\bar{\beta}(\exp (y \otimes 1 / m))=$ $\exp (x \otimes 1)=1, \exp (y \otimes 1 / m) \in K_{\mathrm{D}}$. And it is straightforward to see that $q_{\sigma}(\exp (y \otimes 1 / m))=1$, where $q_{\sigma}:\left(S^{1}\right)^{n} \rightarrow\left(S^{1}\right)^{\left|J_{\sigma}\right|}$ is the projection onto the "non-trivial" components; therefore, $\exp (y \otimes 1 / m) \in \Gamma_{\sigma}$ and we may set $\psi\left(x+N_{\sigma}\right)=\exp (y \otimes 1 / m)$.

The map $\psi$ is well defined. Indeed, suppose we choose a different representative $x^{\prime}+N_{\sigma}$ for $x+N_{\sigma}$ and let $y^{\prime}$ denote the corresponding element in $\mathbb{Z}^{I_{\sigma}}$ with $\beta\left(y^{\prime}\right)=m x^{\prime}$. Then there exists a (unique) $\eta \in \mathbb{Z}^{\left|I_{\sigma}\right|}$ satisfying $\beta(\eta)=x-x^{\prime}$ and hence, $\beta_{\sigma}\left(y-y^{\prime}\right)=\beta_{\sigma}(m \eta)$, which shows $y-y^{\prime}=m \eta$. Therefore, $\exp \left(\left(y-y^{\prime}\right) \otimes 1 / m\right)=\exp (\eta \otimes 1)=1$.

We check that $\psi$ is an isomorphism. To check injectivity, suppose $\psi\left(x+N_{\sigma}\right)=\exp (y \otimes 1 / m)=1$, where $y$ and $m$ are as above. Then $y \otimes 1 / m$ lies in the image of $\mathbb{Z}^{\mid I_{\sigma \mid}} \rightarrow \mathbb{Z}^{\left|I_{\sigma}\right|} \otimes \mathbb{R}$, which implies that $m=1$ so that $x \in N_{\sigma}$. To check surjectivity, suppose $\gamma \in \Gamma_{\sigma} \subset\left(S^{1}\right)^{\left|I_{\sigma}\right|}$. Choose an element $v \in \mathbb{Z}^{\left|I_{\sigma}\right|} \otimes \mathbb{R}$ with $\exp (v)=\gamma$ and consider $\left(\beta_{\sigma}\right)_{\mathbb{R}}(v) \in N_{\mathbb{R}}$. Since $\exp \left(\left(\beta_{\sigma}\right)_{\mathbb{R}}(v)\right)=1$, there is an element $x \in N$ 
such that $x \otimes 1=\beta_{\mathbb{R}}(v)$. We now check that $\psi\left(x+N_{\sigma}\right)=\gamma$. Let $m$ be the order of $\gamma$. Then $\exp (m v)=1$ and hence $m v=\xi \otimes 1$ for some $\xi$ in $\mathbb{Z}^{\left|I_{\sigma}\right|}$, and $\beta_{\sigma}(\xi) \otimes 1=\beta_{\mathbb{R}}(m v)=m x \otimes 1$. Therefore, $\beta_{\sigma}(\xi)=m x$, and $\exp (\xi \otimes 1 / m)=\exp (v)=\gamma$, as required.

Remark 2.16. The isomorphism given in Proposition 2.15 is compatible with the one given in Proposition 2.4 for general $\mathbb{Z}$-modules $N$. Indeed, if $N$ is assumed to be free, then we may choose an isomorphism $\widetilde{q}: \mathbb{Z}^{d} \rightarrow N$ and take $R=\widetilde{q}^{-1} \circ \beta_{\sigma}: \mathbb{Z}^{I} \rightarrow \mathbb{Z}^{d}$ in equation (2.3). With this choice, it can be shown that the following diagram commutes.

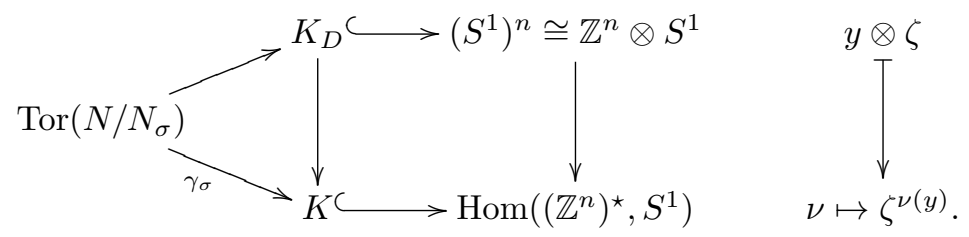

Next, we give an explicit description of the component group $K_{\mathrm{D}} /\left(K_{\mathrm{D}}\right)_{0}$, which models Lemma 2.8 and shows that $\operatorname{coker} \beta \cong$ $K_{\mathrm{D}} /\left(K_{\mathrm{D}}\right)_{0}$.

Define a homomorphism $\varphi: N \rightarrow K_{\mathrm{D}} /\left(K_{\mathrm{D}}\right)_{0}$ as follows. For $x \in N$, choose $y \in \mathbb{Z}^{n} \otimes \mathbb{R}$ with $\beta_{\mathbb{R}}(y)=x \otimes 1$ and consider $\exp (y) \in\left(S^{1}\right)^{n}$. Then $\bar{\beta}(\exp (y))=1$ and thus $\exp (y) \in K_{\mathrm{D}}$. Set $\varphi(x)=\exp (y)\left(K_{\mathrm{D}}\right)_{0}$. The map $\varphi$ is well defined since ker $\beta_{\mathbb{R}}$ is the Lie algebra of $K_{\mathrm{D}}$, which exponentiates onto $\left(K_{\mathrm{D}}\right)_{0}$.

The homomorphism $\varphi$ has kernel $\operatorname{im}(\beta)$. Indeed, if $\varphi(x)=\exp (y) \in$ $\left(K_{\mathrm{D}}\right)_{0}$, then there is an element $\zeta \in \operatorname{ker} \beta_{\mathbb{R}}$ with $\exp (y-\zeta)=1$, and thus an integer vector $a \in \mathbb{Z}^{n}$ with $y-\zeta=a \otimes 1$. Since $N \rightarrow N \otimes \mathbb{R}$ is injective $\left(N\right.$ is free), $x \otimes 1=\beta_{\mathbb{R}}(y)=\beta_{\mathbb{R}}(a \otimes 1)=\beta(a) \otimes 1$ implies $\beta(a)=x$ and hence $\operatorname{ker} \varphi \subset \operatorname{im}(\beta)$. Finally, if $x=\beta(a)$ for some $a \in \mathbb{Z}^{n}$, then we may choose $y=a \otimes 1$ to compute $\varphi(x)=\exp (y)\left(K_{\mathrm{D}}\right)_{0}$ to see that $\exp (y)=1$, showing $\operatorname{im} \beta \subset \operatorname{ker} \varphi$. Hence, we obtain the following:

Proposition 2.17. Suppose $N$ is torsion free. The map $\varphi: N \rightarrow$ $K_{\mathrm{D}} /\left(K_{\mathrm{D}}\right)_{0}$ defined above descends to an isomorphism $\bar{\varphi}: \operatorname{coker} \beta \stackrel{\cong}{\rightrightarrows}$ $K_{\mathrm{D}} /\left(K_{\mathrm{D}}\right)_{0}$. 
Remark 2.18. The above arguments are essentially applications of the Snake lemma (and parts of its proof). For example, Proposition 2.17 above follows part of the proof of the Snake lemma for the diagram of short exact sequences,

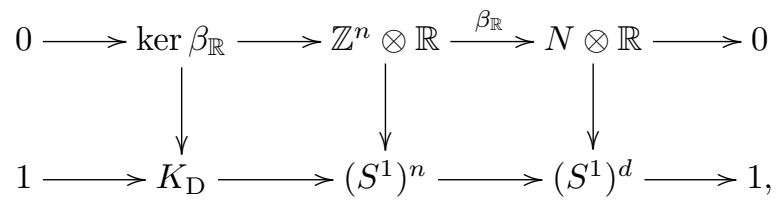

and the map $\varphi$ is the connecting homomorphism.

3. Weighted and fake weighted projective stacks. In this section, we interpret the results in Section 2 for an important class of toric DM stacks known as weighted projective stacks. In subsection 3.1 we identify those toric DM stacks that are equivalent to weighted projective stacks in terms of their stacky fan data. Then in subsection 3.2 we generalize our considerations to fake weighted projective stacks.

3.1. Weighted projective stacks. As mentioned previously, weighted projective stacks are an important class of examples and have been studied extensively both as stacks and as orbifolds. In this section, we characterize those stacky polytopes corresponding to weighted projective stacks.

Definition 3.1. For positive integers $b_{0}, \ldots, b_{d}$, let $\mathbb{T}$ act on $\mathbb{C}^{d+1} \backslash$ $\{0\}$ by $t \cdot\left(z_{0}, \ldots, z_{d}\right)=\left(t^{b_{0}} z_{0}, \ldots, t^{b_{d}} z_{d}\right)$. The resulting quotient stack $\left[\left(\mathbb{C}^{d+1} \backslash\{0\}\right) / \mathbb{T}\right]$ is called a weighted projective stack, denoted $\mathcal{P}\left(b_{0}, \ldots, b_{d}\right)$.

In particular, a weighted projective stack is a quotient by a connected one-dimensional Abelian Lie group action but the action need not be effective.

Recall that to each $\mathcal{P}\left(b_{0}, \ldots, b_{d}\right)$ there is an associated stacky polytope $(N, \Delta, \beta)$ with $\mathrm{DG}(\beta) \cong \mathbb{Z}$ (see, [26, Example 21]) whose associated toric DM stack is equivalent to $\mathcal{P}\left(b_{0}, \ldots, b_{d}\right)$. Proposition 3.2 below shows that the toric DM stack corresponding to any stacky polytope satisfying $\operatorname{DG}(\beta) \cong \mathbb{Z}$ results in a weighted projective stack. 
Proposition 3.2. Let $(N, \Delta, \beta)$ be a stacky polytope, and let $\Sigma(\Delta)$ be the dual fan to $\Delta$. The associated toric DM stack $\mathcal{X}(N, \Sigma(\Delta), \beta)$ is a weighted projective stack $\mathcal{P}\left(b_{0}, \ldots, b_{d}\right)$ if and only if $\mathrm{DG}(\beta) \cong \mathbb{Z}$. In this case, the polytope $\Delta$ is a simplex, and the weights are determined by the condition that $\left(b_{0}, \ldots, b_{d}\right)$ generates $\operatorname{ker} \beta \subset \mathbb{Z}^{d+1}$.

Remark 3.3. Let $(N, \Delta, \beta)$ be a stacky polytope satisfying the condition $\operatorname{DG}(\beta) \cong \mathbb{Z}$. By Lemma 2.8 , it follows that $\beta$ must be surjective. Additionally, the torsion submodule $\operatorname{Tor}(N)$ of $N$ must be cyclic, and the proof of Proposition 3.2 shows that the order of $\operatorname{Tor}(N)$ is $g=\operatorname{gcd}\left(b_{0}, \ldots, b_{d}\right)$.

Proof of Proposition 3.2. Since $\mathrm{DG}(\beta) \cong \mathbb{Z}$, the homomorphism $\beta$ of the stacky polytope $(N, \Delta, \beta)$ must have domain $\mathbb{Z}^{d+1}$ where (as usual) $\operatorname{rank} N=d$. That is the polytope $\Delta$ has $d+1$ facets, and is therefore a simplex. Hence, $V(J(\Sigma(\Delta)))=\{0\}$ and $Z_{\Sigma(\Delta)}=\mathbb{C}^{d+1} \backslash\{0\}$.

We determine the $G$-action on $\mathbb{C}^{d+1} \backslash\{0\}$. Recall that the action is determined by applying $\operatorname{Hom}(-, \mathbb{T})$ to the map $\beta^{\vee}:\left(\mathbb{Z}^{d+1}\right)^{\star} \rightarrow \operatorname{DG}(\beta)$, obtaining a homomorphism $G \rightarrow \mathbb{T}^{d+1}$. We set out to determine $\beta^{\vee}$.

We first show that $\mathrm{DG}(\beta) \cong(\operatorname{ker} \beta)^{\star}$. Let $0 \rightarrow \mathbb{Z}^{\ell} \stackrel{Q}{\rightarrow} \mathbb{Z}^{d+\ell} \rightarrow N \rightarrow 0$ be a free resolution of $N$, and let $B: \mathbb{Z}^{d+1} \rightarrow \mathbb{Z}^{d+\ell}$ denote a lift of $\beta$. Then $\operatorname{DG}(\beta)=\operatorname{coker}\left[\begin{array}{ll}B & Q\end{array}\right]^{*}$. Similar to the proof of $[\mathbf{5}$, Proposition $2.2]$, an application of the Snake lemma to the diagram

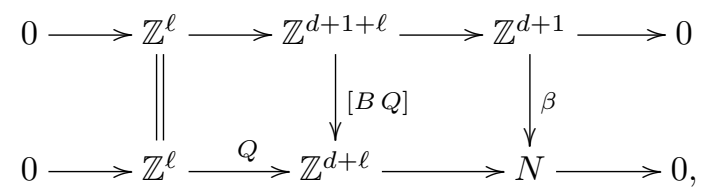

shows that $\left[\begin{array}{ll}B & Q\end{array}\right]$ and $\beta$ have isomorphic cokernels, which are trivial by assumption, as well as isomorphic kernels. It follows that coker $\left[\begin{array}{ll}B & Q\end{array}\right]^{\star} \cong(\operatorname{ker} \beta)^{\star}$.

Since $\beta: \mathbb{Z}^{d+1} \rightarrow N$ has a finite cokernel, it follows that there is an exact sequence

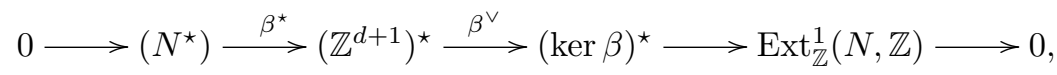


obtained by the identification $\operatorname{DG}(\beta) \cong(\operatorname{ker} \beta)^{\star}$, where exactness at $\operatorname{Ext}_{\mathbb{Z}}^{1}(N, \mathbb{Z})$ follows from the fact that $\operatorname{Ext}_{\mathbb{Z}}^{1}(N, \mathbb{Z})$ is the first right derived functor of $\operatorname{Hom}(-, \mathbb{Z})$ (and $N$ is finitely generated).

Since $\Delta$ is a simplex, we can find positive integers $b_{0}, \ldots, b_{d}$ such that

$$
\sum_{j=0}^{d} b_{j} \beta\left(e_{j}\right) \otimes 1=0,
$$

where $\left\{e_{0}, \ldots e_{d}\right\}$ is the standard basis for $\mathbb{Z}^{d+1}$. Without loss of generality, assume that $b=\left(b_{0}, \ldots, b_{d}\right)$ generates $\operatorname{ker} \beta$, which we now identify with $\mathbb{Z}$ according to $b \leftrightarrow 1$. The resulting identification $(\operatorname{ker} \beta)^{\star} \cong \mathbb{Z}^{\star}$ in equation (3.1) gives $\beta^{\vee}$ the matrix representation $\left[b_{0} \cdots b_{d}\right]$. It follows that $\beta^{\vee}$ induces the homomorphism $\mathbb{T} \rightarrow \mathbb{T}^{d+1}$, $t \mapsto\left(t^{b_{0}}, \ldots, t^{b_{d}}\right)$, which completes the proof.

3.2. Fake weighted projective stacks. Analogous to the fake weighted projective spaces, in this section we consider a fake weighted projective stack, a stack quotient $\mathcal{W} / \Lambda$ where $\Lambda$ is a finite Abelian group acting (in the sense of group actions on stacks $[\mathbf{1 9}, \mathbf{2 5}]$ ) on a weighted projective stack $\mathcal{W}=\mathcal{P}\left(b_{0}, \ldots, b_{d}\right)$. Specifically, we characterize fake weighted projective stacks in terms of their associated combinatorial data.

By Proposition 3.2, the toric DM stack associated to a stacky fan $(N, \Sigma, \beta)$ satisfying $\mathrm{DG}(\beta) \cong \mathbb{Z}$ is a weighted projective stack. If we require only that $\operatorname{rank} \mathrm{DG}(\beta)=1$ (i.e., allowing $\mathrm{DG}(\beta)$ with torsion), the next proposition shows that the resulting toric DM stack is a fake weighted projective stack (compare with [2, Lemma 2.11]). Note that the proof of the implication (i) $\Rightarrow$ (ii) of the proposition relies more heavily on the language of stacks, which in the interest of brevity will not be reviewed. The reader may wish to consult the indicated references.

Proposition 3.4. Let $(N, \Delta, \beta)$ be a stacky polytope, and let $\Sigma(\Delta)$ be the dual fan to $\Delta$. The following statements are equivalent:

(i) the associated toric $\mathrm{DM}$ stack $\mathcal{X}(N, \Sigma(\Delta), \beta)$ is equivalent to a fake weighted projective stack;

(ii) $\operatorname{rank} \mathrm{DG}(\beta)=1$, i.e., $\operatorname{dim} G=1$;

(iii) $\Delta$ is a simplex. 
Under any of the above conditions, $\mathcal{X}(N, \Sigma(\Delta), \beta) \cong \mathcal{P}\left(b_{0}, \ldots, b_{d}\right) / \Lambda$, where the weights are determined by the condition that $\left(b_{0}, \ldots, b_{d}\right)$ generates $\operatorname{ker} \beta \subset \mathbb{Z}^{d+1}$.

Proof. The equivalence of (ii) and (iii) is immediate. Suppose $(N, \Delta, \beta)$ is a stacky polytope with $\operatorname{rank} \mathrm{DG}(\beta)=1$. By [19], $\mathcal{X}(N, \Sigma(\Delta), \beta)=\left[Z_{\Sigma} / G\right] \cong\left[Z_{\Sigma} / G_{0}\right] / \Lambda$, where $\Lambda=G / G_{0}$ and $G_{0}$ denotes the connected component of the identity element in $G$. By Proposition 2.11, $\left[Z_{\Sigma} / G_{0}\right]$ is the toric DM stack associated to the stacky fan $\left(N_{0}, \Sigma(\Delta), \beta_{0}\right)$, which satisfies $\operatorname{DG}\left(\beta_{0}\right) \cong \mathbb{Z}$ and is thus a weighted projective stack, by Proposition 3.2.

Conversely, suppose $\mathcal{X}(N, \Sigma(\Delta), \beta)$ is equivalent to a fake weighted projective stack $\mathcal{W} / \Lambda$ with $\mathcal{W}=\mathcal{P}\left(b_{0}, \ldots, b_{d}\right)$. We shall verify below that the quotient map of stacks $\mathcal{W} \rightarrow \mathcal{W} / \Lambda$ is a covering projection, i.e., a representable map of stacks such that every representative is a covering projection (see, [23]). Since $\mathcal{W}$ has a trivial (stacky) fundamental group, $\mathcal{W}$ is then the universal cover, which by [14], coincides with $\mathcal{X}\left(N_{0}, \Sigma_{0}, \beta_{0}\right)$. Therefore, rank $\mathrm{DG}(\beta)=\operatorname{rank} \mathrm{DG}\left(\beta_{0}\right)=$ 1 by Proposition 3.2. The statement about the weights is also a direct consequence of Proposition 3.2.

To see that $p: \mathcal{W} \rightarrow \mathcal{W} / \Lambda$ is a covering projection, it is enough to show that the base extension of $p$ along a presentation (also called chart or atlas) is a covering projection of topological spaces (since coverings are invariant under base change and local on the target, see, [23, Example 4.6]). Choose a $\Lambda$-atlas $X_{1} \rightrightarrows X_{0}$ for $\mathcal{W}$ with $\Lambda$ acting freely on $X_{1}$ and $X_{0}$ (see, [19]). Then $X_{1} / \Lambda \rightrightarrows X_{0} / \Lambda$ is an atlas for the quotient $\mathcal{W} / \Lambda$. It is straightforward to check that $X_{0} / \Lambda \times_{\mathcal{W} / \Lambda} \mathcal{W} \cong X_{0}$ (e.g., see the discussion of 2-fiber products in [23, Section 9]), and hence the base extension of $p$ is $X_{0} \rightarrow X_{0} / \Lambda$, which is a covering projection.

4. Labeled sheared simplices. We now introduce labeled sheared simplices, a natural family of stacky polytopes that includes many (but not all) stacky polytopes associated with weighted and fake weighted projective stacks. We begin with a definition.

Definition 4.1. Let $\mathbf{a}=\left(a_{1}, \ldots, a_{d}\right) \in \mathbb{Z}^{d}$ be a primitive vector in the positive orthant and $\left\{\epsilon_{i}\right\}$ the standard basis of $\mathbb{R}^{d}$. The sheared 
simplex $\Delta(\mathbf{a})$ is the convex hull of the origin together with the points $\operatorname{lcm}(\mathbf{a}) / a_{j} \epsilon_{j}, j=1, \ldots, d$, in $\mathbb{R}^{d}$. Given positive integers $m_{0}, \ldots, m_{d}$, we define a labeled sheared simplex as the stacky polytope $\left(\mathbb{Z}^{d}, \Delta(\mathbf{a}), \beta\right)$, where the homomorphism $\beta: \mathbb{Z}^{d+1} \rightarrow \mathbb{Z}^{d}$ is given by $\beta\left(e_{0}\right)=-m_{0} \mathbf{a}$ and $\beta\left(e_{j}\right)=m_{j} \epsilon_{j}(j=1, \ldots, d)$, where $e_{0}, \ldots, e_{d}$ denote the standard basis vectors for $\mathbb{Z}^{d+1}$.

We first note that Proposition 3.4 immediately implies that, for a labeled sheared simplex $\left(\mathbb{Z}^{d}, \Delta(\mathbf{a}), \beta\right)$, the associated toric DM stack $\left[Z_{\Sigma(\Delta)} / G\right]$ is indeed a fake weighted projective stack. The concrete combinatorial description of these simplices provide an interesting explicit class of examples of fake projective stacks to study.

Since all labeled sheared simplices give rise to fake weighted projective stacks, we first identify, in subsection 4.1, precisely which labeled sheared simplices correspond to weighted projective stacks. We analyze the fake weighted projective stacks arising from labeled sheared simplices more generally in subsection 4.2, and finally, we give a detailed analysis of two-dimensional labeled sheared simplices in subsection 4.3.

\subsection{Labeled sheared simplices corresponding to weighted} projective stacks. The main result of this section is Proposition 4.4, in which we identify those labeled sheared simplices that give rise to a weighted projective stack. By Proposition 3.2, this involves translating the condition $\mathrm{DG}(\beta) \cong \mathbb{Z}$ in terms of the labels $\left\{m_{0}, \ldots, m_{d}\right\}$ and the vector a. Since $\operatorname{rank} \operatorname{DG}(\beta)=1, \operatorname{DG}(\beta) \cong \mathbb{Z}$ if and only if $G$ is connected; therefore, we begin with the following lemma.

Lemma 4.2. Let $\left(\mathbb{Z}^{d}, \Delta(\mathbf{a}), \beta\right)$ be a labeled sheared simplex with labels $\left\{m_{0}, \ldots, m_{d}\right\}$, and let $\left[Z_{\Sigma} / G\right]$ be its associated toric DM stack. If $G_{0} \subset G$ denotes the connected component of the identity element, then

$$
G / G_{0} \cong\left[\bigoplus_{i=0}^{d} \mathbb{Z} / m_{i} \mathbb{Z}\right] /\left\langle\left(1 \bmod m_{0}, a_{1} \bmod m_{1}, \ldots, a_{d} \bmod m_{d}\right)\right\rangle
$$

Example 4.3. Consider the toric DM stack from Example 1.5, the labeled line segment with labels $r$ and $s$, where it was seen directly that $G \cong \mathbb{T} \times \mu_{g}$, where $g=\operatorname{gcd}(r, s)$ and $\mu_{g} \subset \mathbb{T}$ is the cyclic group of $g$ th roots of unity. Hence, $G / G_{0} \cong \mu_{g}$. This can be seen from 
Lemma 4.2 as well, since $G / G_{0} \cong(\mathbb{Z} / r \mathbb{Z} \oplus \mathbb{Z} / s \mathbb{Z}) / H$ where $H$ is the subgroup generated by $(1,1)$.

Proof of Lemma 4.2. By Lemma 2.8, $G / G_{0} \cong \operatorname{coker} \beta$, which we now compute. Consider the commutative diagram of short exact sequences:

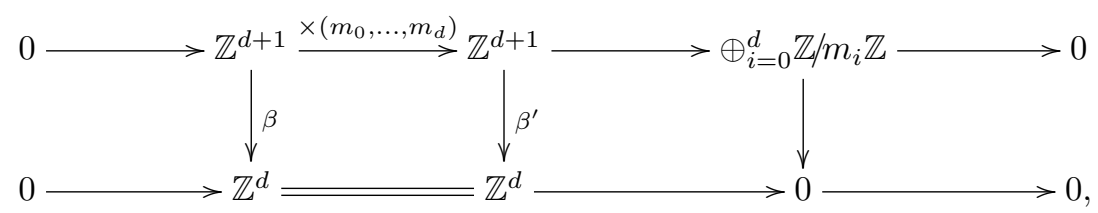

where $\beta^{\prime}$ may be written as the matrix

$$
\left[\begin{array}{cccc}
-a_{1} & 1 & & 0 \\
\vdots & & \ddots & \\
-a_{d} & 0 & & 1
\end{array}\right] .
$$

Applying the Snake lemma gives the exact sequence

$$
0 \longrightarrow \operatorname{ker} \beta \longrightarrow \operatorname{ker} \beta^{\prime} \longrightarrow \bigoplus_{i=0}^{d} \mathbb{Z} / m_{i} \mathbb{Z} \longrightarrow \operatorname{coker} \beta \longrightarrow 0
$$

Since $\operatorname{ker} \beta^{\prime} \cong \mathbb{Z}$ is generated by $\left(1, a_{1}, \ldots, a_{d}\right) \in \mathbb{Z}^{d+1}$, the second map in the sequence above sends the generator to $\left(1 \bmod m_{0}, a_{1} \bmod \right.$ $\left.m_{1}, \ldots, a_{d} \bmod m_{d}\right)$. The result follows.

Proposition 4.4. Let $\left(\mathbb{Z}^{d}, \Delta(\boldsymbol{a}), \beta\right)$ be a labeled sheared simplex with labels $\left\{m_{0}, \ldots, m_{d}\right\}$ and $\mathbf{a}=\left(a_{1}, \ldots, a_{d}\right)$. Let $M=m_{0} m_{1} \cdots m_{d}$. The following conditions are equivalent:

(i) $\operatorname{DG}(\beta) \cong \mathbb{Z}$;

(ii) $\mathcal{X}\left(\mathbb{Z}^{d}, \Delta(\mathbf{a}), \beta\right)$ is equivalent to a weighted projective stack;

(iii) $\operatorname{gcd}\left(\frac{M}{m_{0}}, \frac{M a_{1}}{m_{1}}, \ldots, \frac{M a_{d}}{m_{d}}\right)=1$;

(iv) $\operatorname{gcd}\left(m_{i}, m_{j}\right)=1$ for all $i \neq j$ in $\{0, \ldots, d\}$, and $\operatorname{gcd}\left(a_{i}, m_{i}\right)=1$, for all $i$ in $\{1, \ldots, d\}$.

In addition, if one of the above holds, then

$$
\mathcal{X}\left(\mathbb{Z}^{d}, \Delta(\mathbf{a}), \beta\right)=\mathcal{P}\left(\frac{M}{m_{0}}, \frac{M a_{1}}{m_{1}}, \ldots, \frac{M a_{d}}{m_{d}}\right) .
$$


Proof. The equivalence of conditions (i) and (ii) is part of Proposition 3.2 , which also gives the identification of the weights.

Let $\left(\mathbb{Z}^{d}, \Delta(\mathbf{a}), \beta\right)$ be a labeled sheared simplex, with labels $\left\{m_{0}, \ldots\right.$, $\left.m_{d}\right\}$ and $\mathbf{a}=\left(a_{1}, \ldots, a_{d}\right)$. Since the rank of $\operatorname{DG}(\beta)$ is 1 , by Lemma 2.8, condition (i) is equivalent to coker $\beta=0$. By Lemma 4.2 , $\operatorname{coker} \beta=0$ if and only if

$$
H=\left\langle\left(1 \bmod m_{0}, a_{1} \bmod m_{1}, \ldots, a_{d} \bmod m_{d}\right)\right\rangle
$$

is equal to $\oplus_{i} \mathbb{Z} / m_{i} \mathbb{Z}$, or equivalently if $H$ is cyclic of order $M=$ $m_{0} m_{1} \cdots m_{d}$. If (iv) holds, this is immediate.

Conversely, suppose $H$ is cyclic of order $M$, and hence equals $\oplus_{i} \mathbb{Z} / m_{i} \mathbb{Z}$. Therefore, $\operatorname{gcd}\left(m_{i}, m_{j}\right)=1$ for $i \neq j$. Consider the isomorphism $\oplus_{i} \mathbb{Z} / m_{i} \mathbb{Z} \rightarrow \mathbb{Z} / M \mathbb{Z}$ defined by sending $f_{i} \mapsto M / m_{i}$, where $f_{i}$ denotes the element whose only non-zero component is $1 \mathrm{mod}$ $m_{i}$ in the $i$ th component. Under this isomorphism, the generator of $H$ is sent to $b=\left(M / m_{0}+M a_{1} / m_{1}+\cdots+M a_{d} / m_{d}\right) \bmod M$, which has order $M / \operatorname{gcd}(M, b)=M$ since $H$ has order $M$. Therefore, $\operatorname{gcd}(M, b)=1$, and hence $\operatorname{gcd}\left(m_{j}, a_{j}\right)=1$, which shows (iv).

The equivalence of conditions (iii) and (iv) is straightforward to verify.

Proposition 4.5 below describes the isotropy groups for weighted projective stacks that correspond to labeled sheared simplices. For a weighted projective stack $\mathcal{P}\left(b_{0}, \ldots, b_{d}\right)$, the resulting isotropy groups are straightforward to compute directly from the defining action of $\mathbb{T}$. Namely, the isotropy of a point $z \in \mathbb{C}^{d+1} \backslash\{0\}$ is easily seen to be cyclic of order $\operatorname{gcd}\left(b_{j}: z_{j} \neq 0\right)$. For those weighted projective stacks arising from labeled sheared simplices, we may use Proposition 4.4 to express this in terms of the labels $\left\{m_{0}, \ldots, m_{d}\right\}$ and $\mathbf{a}$.

Proposition 4.5. Suppose that $\left(\mathbb{Z}^{d}, \Delta(\boldsymbol{a}), \beta\right)$ is a labeled sheared simplex with labels $\left\{m_{0}, m_{1}, \ldots, m_{d}\right\}$ that corresponds to a weighted projective stack. Let $z=\left(z_{0}, \ldots, z_{d}\right) \in \mathbb{C}^{d+1} \backslash\{0\}$, and set $d_{z}=\operatorname{gcd}\left(a_{j}\right.$ : $\left.z_{j} \neq 0\right)$, where $a_{0}$ is set to 1 . If $z_{0} \neq 0$, then $\operatorname{Stab}(z) \cong \bigoplus_{i \in I_{z}} \mathbb{Z} / m_{i} \mathbb{Z}$. If $z_{0}=0$, then

$$
\operatorname{Stab}(z) \cong \mathbb{Z} /\left(m_{z} d_{z}\right) \mathbb{Z}, \quad \text { where } m_{z}=\prod_{i \in I_{z}} m_{i} .
$$


The stacky polytope $\left(\mathbb{Z}^{2}, \Delta, \beta\right)$ introduced in Example 1.3 is a labeled sheared simplex $\Delta(1,1)$ with labels $\{2,3,5\}$. By Proposition 4.5, the associated toric DM stack is a weighted projective stack; therefore, we may use Proposition 4.5 to reproduce the calculation in Example 2.3.

Remark 4.6. The results in this section depend only on the dual fan $\Sigma(\Delta)$, which consists of all cones $\sigma$ generated by any subset of the ray generators $\left\{\mathbf{a}, \epsilon_{1}, \ldots, \epsilon_{d}\right\}$.

4.2. On fake weighted projective stacks arising from labeled sheared simplices. In this section, we study labeled sheared simplices more generally. Specifically, we analyze the isotropy groups of the corresponding fake weighted projective stacks in Proposition 4.7, and we characterize the labeled sheared simplices giving rise to global quotient stacks in Proposition 4.8.

We begin with a result on isotropy groups.

Proposition 4.7. Let $\left(\mathbb{Z}^{d}, \Delta(\mathbf{a}), \beta\right)$ be a labeled sheared simplex with labels $\left\{m_{0}, \ldots, m_{d}\right\}$, and set $a_{0}=1$. Let $z=\left(z_{0}, \ldots, z_{d}\right) \in \mathbb{C}^{d} \backslash\{0\}$, and set $d_{z}=\operatorname{gcd}\left(a_{j}: z_{j} \neq 0\right)$. The isotropy group $\operatorname{Stab}(z)$ is an extension as follows:

$$
0 \longrightarrow \bigoplus_{i \in I_{z}} \mathbb{Z} / m_{i} \mathbb{Z} \longrightarrow \operatorname{Stab}(z) \longrightarrow \mathbb{Z} / d_{z} \mathbb{Z} \longrightarrow 0 .
$$

Proof. Recall that by Theorem 2.2, the isotropy group $\operatorname{Stab}(z)$ is isomorphic to the torsion submodule of $N / N_{z}$. Form the matrix $B_{z}$ by deleting the $i$ th column of $\beta$ whenever $z_{i} \neq 0$ Viewing $B_{z}$ as a homomorphism $\mathbb{Z}^{\left|I_{z}\right|} \rightarrow \mathbb{Z}^{d}$ realizes $N / N_{z}=\operatorname{coker} B_{z}$. We compute the torsion submodule of coker $B_{z}$ next.

If $z_{0} \neq 0$, then one may readily see that $\operatorname{Tor}\left(N / N_{z}\right) \cong \bigoplus_{i \in I_{z}} \mathbb{Z} / m_{i} \mathbb{Z}$. It remains to consider the case where $z_{0}=0$, i.e., with corresponding matrix $B_{z}$ containing the first column of $\beta$.

To begin, observe that

$$
\beta: \mathbb{Z}^{d+1} \longrightarrow N=\mathbb{Z}^{d}
$$


factors as the composition

$$
\mathbb{Z}^{d+1} \stackrel{L}{\longrightarrow} \mathbb{Z}^{d+1} \stackrel{\beta^{\prime}}{\longrightarrow} \mathbb{Z}^{d}
$$

where

$$
L=\left[\begin{array}{ccc}
m_{0} & & 0 \\
& \ddots & \\
0 & & m_{d}
\end{array}\right] \text { and } \beta^{\prime}=\left[\begin{array}{cccc}
-a_{1} & 1 & & 0 \\
\vdots & & \ddots & \\
-a_{d} & 0 & & 1
\end{array}\right]
$$

Accordingly, we may factor $B_{z}$ as a composition $\mathbb{Z}^{\left|I_{z}\right|} \stackrel{L_{z}}{\rightarrow} \mathbb{Z}^{\left|I_{z}\right|} \stackrel{B_{z}^{\prime}}{\rightarrow} \mathbb{Z}^{d}$, where $B_{z}^{\prime}$ is a matrix whose first column is the first column of $\beta^{\prime}$ and whose other columns are standard basis vectors $e_{i} \in \mathbb{Z}^{d}$ for $i \neq 0$ in $I_{z}$. This factorization yields the following diagram of short exact sequences:

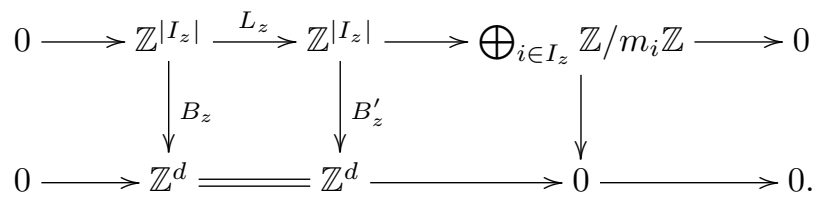

Applying the Snake lemma (and observing that $B_{z}^{\prime}$ is injective) yields the exact sequence,

$$
0 \longrightarrow \bigoplus_{i \in I_{z}} \mathbb{Z} / m_{i} \mathbb{Z} \longrightarrow \operatorname{coker} B_{z} \longrightarrow \operatorname{coker} B_{z}^{\prime} \longrightarrow 0
$$

Let $d_{z}=\operatorname{gcd}\left\{a_{i}: i \notin I_{z}\right\}$. Then $B_{z}^{\prime}$ is row equivalent to the matrix $C_{z}^{\prime}$ obtained from $B_{z}^{\prime}$ by replacing all $a_{i}$ with $i \notin I_{z}$ with 0's except for one which is replaced with $d_{z}$. It follows that coker $B_{z}^{\prime} \cong$ $\mathbb{Z}^{d-\left|I_{z}\right|} \oplus \mathbb{Z} / d_{z} \mathbb{Z}$. Moreover, we may describe the generators of the free summand as follows. Let $E_{z}: \mathbb{Z}^{d} \rightarrow \mathbb{Z}^{d}$ denote the invertible homomorphism defined by $C_{z}^{\prime}=E_{z} B_{z}^{\prime}$. Then the free summand is generated by the images of the $E^{-1}\left(e_{i}\right), i \notin I_{z}$, in $\mathbb{Z}^{d} / \mathrm{im} B_{z}^{\prime}$. It follows that the $E^{-1}\left(e_{i}\right), i \notin I_{z}$, must generate a free submodule in coker $B_{z}$, which maps isomorphically onto the free summand of coker $B_{z}^{\prime}$. Thus, we may pass to torsion submodules in equation (4.1):

$$
0 \longrightarrow \bigoplus_{i \in I_{z}} \mathbb{Z} / m_{i} \mathbb{Z} \longrightarrow \operatorname{Tor}\left(\operatorname{coker} B_{z}\right) \longrightarrow \mathbb{Z} / d_{z} \mathbb{Z} \longrightarrow 0
$$

As we will see in subsection 4.3, the group extension in Proposition 4.7 can be non-trivial. For example, consider the labeled sheared 
simplex $\Delta(1,2)$ with labels $m_{0}=2, m_{1}=4, m_{2}=1$. By Proposition 4.10 in subsection 4.3 (or by direct calculation), the isotropy for points of the form $(0,0, z) \in Z_{\Sigma(\Delta)}, z \neq 0$, is $\mathbb{Z} / 2 \mathbb{Z} \oplus \mathbb{Z} / 8 \mathbb{Z}$, a non-trivial extension of $\mathbb{Z} / 2 \mathbb{Z}$ by $\mathbb{Z} / 2 \mathbb{Z} \oplus \mathbb{Z} / 4 \mathbb{Z}$.

Using Corollary 2.12, we may also characterize those labeled sheared simplices yielding global quotient stacks. Note that since the toric DM stack constructed from a labeled sheared simplex is a fake weighted projective stack, it is immediate that a global quotient in this case must then be a quotient of smooth projective stack $\mathcal{P}^{d}$.

Proposition 4.8. Let $\left(\mathbb{Z}^{d}, \Delta(\mathbf{a}), \beta\right)$ be a labeled sheared simplex with labels $\left\{m_{0}, \ldots, m_{d}\right\}$. The toric $\mathrm{DM}$ stack $\mathcal{X}\left(\mathbb{Z}^{d}, \Delta(\mathbf{a}), \beta\right)$ is equivalent to a global quotient if and only if $m_{i}=m_{0} a_{i}$ for all $i=1 \ldots, d$.

Proof. We apply Corollary 2.12 and [14, Theorem 4.4]. Let $\sigma_{j}$ be the maximal cone generated by the rays $\left\{\rho_{0}, \ldots, \widehat{\rho_{j}}, \ldots, \rho_{d}\right\}$, where signifies omission from the list. Then

$$
N_{\sigma_{0}}^{\prime}=\operatorname{span}\left\{m_{1} e_{1}, \ldots, m_{d} e_{d}\right\}
$$

and

$$
N_{\sigma_{j}}^{\prime}=\operatorname{span}\left\{m_{0} \sum_{i=1}^{d} a_{i} e_{i}, m_{1} e_{1}, \ldots, \widehat{m_{j} e_{j}}, \ldots, m_{d} e_{d}\right\}
$$

Observe that if $m_{0} a_{i}=m_{i}$ for all $i$, then it is clear that $N^{\prime}=N_{\sigma_{j}}^{\prime}$ for all $j=0 \ldots, d$.

To prove the converse, suppose $N^{\prime}=N_{\sigma_{j}}^{\prime}$ for all $j=0, \ldots d$. For $j=0$, this implies that there exist $\alpha_{1}, \ldots, \alpha_{d} \in \mathbb{Z}$ such that

$$
\sum_{i=1}^{d}\left(m_{0} a_{i}-\alpha_{i} m_{i}\right) e_{i}=0
$$

and thus $m_{i} \mid m_{0} a_{i}$ for $i=1, \ldots, d$. Similarly, for $j=1, \ldots, d$, we see that there exist $\gamma_{0}, \ldots, \widehat{\gamma_{j}}, \ldots, \gamma_{d} \in \mathbb{Z}$ such that

$$
m_{j} e_{j}=\gamma_{0} m_{0} \sum_{i=1}^{d} a_{i} e_{i}+\sum_{i \neq j} \gamma_{i} m_{i} e_{i}
$$


or, equivalently,

$$
\sum_{i \neq j}\left(\gamma_{0} m_{0} a_{i}+\gamma_{i} m_{i}\right) e_{i}+\left(\gamma_{0} m_{0} a_{j}-m_{j}\right) e_{j}=0 .
$$

Therefore, $m_{0} a_{j} \mid m_{j}$ for $j=1, \ldots, d$, whence $m_{0} a_{i}=m_{i}$ for all $i$.

4.3. Two-dimensional labeled sheared simplices. In this final section, we illustrate the results in previous sections by considering the class of toric DM stacks arising from two-dimensional labeled sheared simplices. As a consequence of Theorem 2.2, we can now determine the isotropy groups corresponding to a labeled sheared simplex in the plane (see also Remark 2.5).

Let $\mathbf{a}=\left(a_{1}, a_{2}\right)$ be a primitive vector in the positive quadrant, and suppose $\left(\mathbb{Z}^{2}, \Delta(\mathbf{a}), \beta\right)$ is a labeled sheared simplex with labels $\left\{m_{0}, m_{1}, m_{2}\right\}$. Explicitly, $\Delta(\mathbf{a})$ is the convex hull of the origin together with $\left(a_{2}, 0\right)$ and $\left(0, a_{1}\right)$, with assigned labels $m_{1}$ to the edge along the $y$-axis, $m_{2}$ to the edge along the $x$-axis, and $m_{0}$ to the remaining edge (see Figure 4).

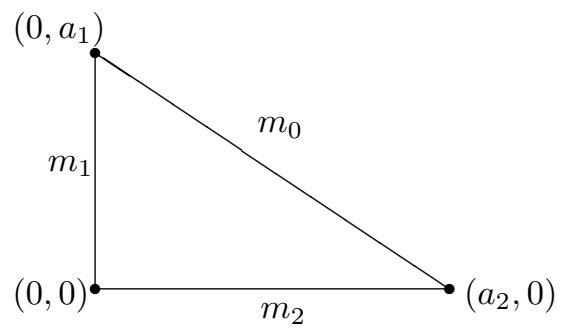

FIgURE 4. A labeled sheared simplex in the plane.

The isotropy for points $\left(z_{0}, 0,0\right) \in Z_{\Sigma}=\mathbb{C}^{3} \backslash\{0\}$ with $z_{0} \neq 0$ is easily seen to be $\mathbb{Z} / m_{1} \mathbb{Z} \oplus \mathbb{Z} / m_{2} \mathbb{Z}$ (by Proposition 4.7 ). For points of the form $z=\left(0,0, z_{2}\right)$ with $z_{2} \neq 0$, i.e., for points corresponding to the vertex $\left(0, a_{1}\right)$, we shall describe the isotropy $\operatorname{Stab}(z)$ below. (The isotropy for points of the form $z=\left(0, z_{1}, 0\right)$ with $z_{1} \neq 0$ can be obtained by exchanging the indices 1 and 2.) 
We begin with a lemma describing the Smith normal form of an integer matrix with exactly one zero entry.

Lemma 4.9. For non-zero $a, b, c \in \mathbb{Z}$, the Smith normal form of $\left[\begin{array}{ll}a & b \\ c & 0\end{array}\right]$ is $\left[\begin{array}{cc}g & 0 \\ 0 & b c / g\end{array}\right]$, where $g=\operatorname{gcd}(a, b, c)$.

Proof. Suppose that $\operatorname{gcd}(a, b)=d$. Then, we claim that there exist $x, y \in \mathbb{Z}$ such that $x a+y b=d$, and $\operatorname{gcd}(x, d)=1$.

To prove this claim, we first note that it is equivalent to the following: Suppose $u, v \in \mathbb{Z}$ are relatively prime. Consider the set of solutions $X=\{x \mid x u+y v=1$ for some $y \in \mathbb{Z}\}$. For any given integer $d$, there is some $x \in X$ so that $\operatorname{gcd}(x, d)=1$.

In this latter formulation, let $d \in \mathbb{Z}$ be given, and suppose that $x_{0}$ is any solution to $x_{0} u+y v=1$. Recall that all solutions are then of the form $x=x_{0}+t v$ with $t \in \mathbb{Z}$. Moreover, $x_{0} u+y v=1$ implies that $\operatorname{gcd}\left(x_{0}, v\right)=1$. Then, showing that there is an $x \in X$ such that $\operatorname{gcd}(x, d)=1$ is equivalent to showing that there is a $t \in \mathbb{Z}$ such that $\operatorname{gcd}\left(x_{0}+t v, d\right)=1$. We will construct such an integer, $t$.

Suppose that $d=p_{1}^{\alpha_{1}} \cdot \ldots \cdot p_{s}^{\alpha_{s}}$ is the prime factorization of $d$. Let $t=\prod p_{i}$ such that $p_{i}$ does not appear in the prime factorization of either $x_{0}$ or $v$. Because $x_{0}$ and $v$ are relatively prime, it follows that, in the sum $x_{0}+t v$, each prime in the factorization of $d$ appears exactly once, that is, each $p_{i}$ divides exactly one of $x_{0}$ or $t v$. Thus, $d$ cannot divide the sum and $\operatorname{gcd}(x, d)=1$.

Therefore, we may find $x$ and $y$ such that $a x+b y=d$ and $\operatorname{gcd}(x, d)=1$. Moreover, since $\operatorname{gcd}(c x, d)=\operatorname{gcd}(c, d)=\operatorname{gcd}(a, b, c)=g$, there exist $p, q \in \mathbb{Z}$ such that $p(c x)+q d=g$. Hence,

$$
\left[\begin{array}{cc}
x & -b / d \\
y & a / d
\end{array}\right], \quad\left[\begin{array}{cc}
q & p \\
-c x / g & d / g
\end{array}\right] \in \mathrm{SL}_{2}(\mathbb{Z})
$$

and

$$
\left[\begin{array}{cc}
q & p \\
-c x / g & d / g
\end{array}\right]\left[\begin{array}{ll}
a & b \\
c & 0
\end{array}\right]\left[\begin{array}{cc}
x & -b / d \\
y & a / d
\end{array}\right]=\left[\begin{array}{cc}
g & -c b p / d \\
0 & -b c / g
\end{array}\right] .
$$

Recall that $d \mid b$ and that $g \mid c$, so $g \mid(c b p / d)$. Thus, by elementary 
column operations

$$
\left[\begin{array}{cc}
g & -c b p / d \\
0 & -b c / g
\end{array}\right] \longleftrightarrow\left[\begin{array}{cc}
g & 0 \\
0 & -b c / g
\end{array}\right]
$$

Finally, note that $g \mid(b c / g)$, so the above is the desired Smith normal form.

We now can give the explicit form of the isotropy groups of a labeled sheared simplex in the plane.

Proposition 4.10. Let $\left(\mathbb{Z}^{2}, \Delta(\mathbf{a}), \beta\right)$ be a labeled sheared simplex with labels $\left\{m_{0}, m_{1}, m_{2}\right\}$ and $\left[Z_{\Sigma} / G\right]$ its corresponding toric DM stack. The isotropy of $z=\left(0,0, z_{2}\right) \in Z_{\Sigma}$ with $z_{2} \neq 0$ is $\operatorname{Stab}(z) \cong \mathbb{Z} / g \mathbb{Z} \oplus$ $\mathbb{Z} /\left(\left(m_{0} m_{1} a_{2}\right) / g\right) \mathbb{Z}$, where $g=\operatorname{gcd}\left(m_{0}, m_{1}\right)$.

Proof. We consider the map $\beta: \mathbb{Z}^{3} \rightarrow \mathbb{Z}^{2}$ given by the matrix

$$
\beta=\left[\begin{array}{ccc}
-m_{0} a_{1} & m_{1} & 0 \\
-m_{0} a_{2} & 0 & m_{2}
\end{array}\right] .
$$

By Lemma 4.9, the Smith normal form of

$$
B_{z}=\left[\begin{array}{cc}
-m_{0} a_{1} & m_{1} \\
-m_{0} a_{2} & 0
\end{array}\right] \quad \text { is } \quad\left[\begin{array}{cc}
g & 0 \\
0 & m_{0} m_{1} a_{2} / g
\end{array}\right]
$$

since $g=\operatorname{gcd}\left(m_{0}, m_{1}\right)=\operatorname{gcd}\left(m_{0} a_{1}, m_{1}, m_{0} a_{2}\right)$, which, by Theorem 2.2, gives the result.

Although Proposition 4.10 gives the general form of the isotropy group of points corresponding to the vertex $\left(0, a_{1}\right)$ of a sheared simplex,

TABLE 1. The isotropy group $\operatorname{Stab}(z)$ corresponding to points of the form $\left(0,0, z_{2}\right)$ with $z_{2} \neq 0$, i.e., corresponding to the vertex $\left(0, a_{1}\right)$ of $\Delta$, for a toric DM stack corresponding to a labeled sheared simplex $\left(\mathbb{Z}^{2}, \Delta(\mathbf{a}), \beta\right)$. Here, $g=\operatorname{gcd}\left(m_{0}, m_{1}\right)$.

\begin{tabular}{|c|c|c|}
\hline Labels & Lengths & Stab $(z)$ \\
\hline \multirow{2}{*}{$m_{0}=m_{1}=m_{2}=1$} & $a_{1}=a_{2}=1$ & $\{1\}$, i.e., smooth \\
\cline { 2 - 3 } & $a_{1}, a_{2}$ arbitrary & $\mathbb{Z} / a_{1} \mathbb{Z}$ \\
\hline \multirow{2}{*}{$m_{0}, m_{1}, m_{2}$ arbitrary } & $a_{1}=a_{2}=1$ & $\mathbb{Z} / m_{0} \mathbb{Z} \oplus \mathbb{Z} / m_{1} \mathbb{Z}$ \\
\cline { 2 - 3 } & $a_{1}, a_{2}$ arbitrary & $\mathbb{Z} / g \mathbb{Z} \oplus \mathbb{Z} /\left(m_{0} m_{1} a_{2} / g\right) \mathbb{Z}$ \\
\hline
\end{tabular}


it can be instructive to consider several special cases to illustrate the interplay of the facet labels and the geometry of the sheared simplex, see Table 1.

Acknowledgments. We would like to thank Yael Karshon, Graham Denham and Jim Lawrence for useful discussions on both toric varieties and convex polytopes.

\section{REFERENCES}

1. J. Alper, A guide to the literature on algebraic stacks, https://maths-people.anu.edu.au/ alperj/papers/stacks-guide.pdf, available from the author's webpage.

2. V. Batyrev and D. Cox, On the Hodge structure of projective hypersurfaces in toric varieties, Duke Math. J. 75 (1994), 293-338.

3. K. Behrend, B. Conrad, D. Edidin, B. Fantechi, W. Fulton, L. Göttssche and A. Kresch, Algebraic stacks, http://www.math.uzh.ch/index.php?pr_vo_det\&key1 $=1287 \&$ key $2=580 \& n o \_$cache $=1$, in progress.

4. S. Boissière, É. Mann and F. Perroni, A model for the orbifold Chow ring of weighted projective spaces, Comm. Alg. 37 (2009), 503-514.

5. L.A. Borisov, L. Chen and G.G. Smith, The orbifold Chow ring of toric Deligne-Mumford stacks, J. Amer. Math. Soc. 18 (2005), 193-215 (electronic).

6. W. Buczyńska, Fake weighted projective space, M.S. thesis, Warsaw University, arXiv:0805.1211 [math.AG].

7. D. Edidin, What is a stack?, Not. Amer. Math. Soc. 50 (2003), 458-459.

8. B. Fantechi, Stacks for everybody, in European congress of mathematics, Vol. I, Progr. Math. 201, Birkhäuser, Basel, 2001.

9. B. Fantechi, E. Mann and F. Nironi, Smooth toric Deligne-Mumford stacks, J. reine angew. Math. 648 (2010), 201-244.

10. W. Fulton, Introduction to toric varieties, Ann. Math. Stud. 131, Princeton University Press, Princeton, NJ, 1993.

11. A. Geraschenko and M. Satriano, Toric stacks I: The theory of stacky fans, Trans. Amer. Math. Soc. 367 (2015), 1033-1071.

12. , Toric stacks II: Intrinsic characterization of toric stacks, Trans. Amer. Math. Soc. 367 (2015), 1073-1094.

13. R. Goldin, T.S. Holm and A. Knutson, Orbifold cohomology of torus quotients, Duke Math. J. 139 (2007), 89-139.

14. M. Harada and D. Krepski, Global quotients among toric Deligne-Mumford stacks, Osaka J. Math. 52 (2014), 236-269.

15. I. Iwanari, Logarithmic geometry, minimal free resolutions and toric algebraic stacks, Publ. Res. Inst. Math. Sci. 45 (2009), 1095-1140. 
16. Y. Jiang, The Chen-Ruan cohomology of weighted projective spaces, Canad. J. Math. 59 (2007), 981-1007.

17. A. Kasprzyk, Bounds on fake weighted projective spaces, Kodai Math. J. 32 (2009), 197-208.

18. E. Lerman, Orbifolds as stacks?, Enseign. Math. 56 (2010), 315-363.

19. E. Lerman and A. Malkin, Hamiltonian group actions on symplectic Deligne-Mumford stacks and toric orbifolds, Adv. Math. 229 (2012), 984-1000.

20. E. Lerman and S. Tolman, Hamiltonian torus actions on symplectic orbifolds and toric varieties, Trans. Amer. Math. Soc. 349 (1997), 4201-4230.

21. E. Mann, Orbifold quantum cohomology of weighted projective spaces, J. Alg. Geom. 17 (2008), 137-166.

22. D. Metzler, Topological and smooth stacks, arXiv:math/0306176 [math.DG].

23. B. Noohi, Foundations of topological stacks I, arXiv:math/0503247 [math.AG].

24. F. Perroni, A note on toric Deligne-Mumford stacks, Tohoku Math. J. 60 (2008), 441-458.

25. M. Romagny, Group actions on stacks and applications, Michigan Math. J. 53 (2005), 209-236.

26. H. Sakai, The symplectic Deligne-Mumford stack associated to a stacky polytope, Results Math. 63 (2013), 903-922.

27. C.A. Weibel, An introduction to homological algebra, Cambr. Stud. Adv. Math. 38, Cambridge University Press, Cambridge, 1994.

Mathematical Sciences MS 3F2, George Mason University, 4400 University Drive, FAIRFAX, VA 22030

Email address: rgoldin@gmu.edu

Department of Mathematics and Statistics, McMaster University, 1280 Main Street West, Hamilton, Ontario L8S4K1, Canada

Email address: Megumi.Harada@math.mcmaster.ca

Naval Surface Warfare Center, Dahlgren Division, Dahlgren, VA 22448

Email address: david.johannsen@navy.mil

Department of Mathematics, University of Manitoba, 342 Machray Hall, Winnipeg, Manitoba R3T 2N2, CANADA

Email address: Derek.Krepski@umanitoba.ca 